Western University

Scholarship@Western

Biology Publications

Biology Department

$10-1-2015$

\title{
An invitation to measure insect cold tolerance: Methods, approaches, and workflow.
}

Brent J Sinclair

bsincla7@uwo.ca

Litza E Coello Alvarado

Laura V Ferguson

Follow this and additional works at: https://ir.lib.uwo.ca/biologypub

Part of the Biology Commons

Citation of this paper:

Sinclair, Brent J; Coello Alvarado, Litza E; and Ferguson, Laura V, "An invitation to measure insect cold tolerance: Methods, approaches, and workflow." (2015). Biology Publications. 68.

https://ir.lib.uwo.ca/biologypub/68 
1 REVIEW

2

3 An invitation to measure insect cold tolerance: methods, approaches, and

4 workflow

5 Brent J. Sinclair*, Litza E. Coello Alvarado \& Laura V. Ferguson

6 Department of Biology, University of Western Ontario, London, ON, Canada

7

8 Address for correspondence: Dept. Biology, University of Western Ontario, London, ON,

$9 \quad$ N6A 5B7, Canada

10 Email: bsincla7@uwo.ca; tel: 519-661-2111 x83138; fax: 519-661-3935

11

12 


\section{Abstract}

14 Insect performance is limited by the temperature of the environment, and in temperate,

15 polar, and alpine regions, the majority of insects must face the challenge of exposure to low

16 temperatures. The physiological response to cold exposure shapes the ability of insects to

17 survive and thrive in these environments, and can be measured, without great technical

18 difficulty, for both basic and applied research. For example, understanding insect cold

19 tolerance allows us to predict the establishment and spread of insect pests and biological

20 control agents. Additionally, the discipline provides the tools for drawing physiological

21 comparisons among groups in wider studies that may not be focused primarily on the

22 ability of insects to survive the cold. Thus, the study of insect cold tolerance is of a broad

23 interest, and several reviews have addressed the theories and advances in the field. Here,

24 however, we aim to clarify and provide rationale for common practices used to study cold

25 tolerance, as a starter's guide for newcomers to the field, students, and those wishing to

26 incorporate cold tolerance into a broader study. We cover the 'tried and true' measures of

27 insect cold tolerance, the equipment necessary for these measurement, and summarize the

28 ecological and biological significance of each. Additionally, we provide a suggested

29 framework and workflow for measuring cold tolerance and low temperature performance in 30 insects.

32 Keywords: chill coma, critical thermal minimum, supercooling point, cold tolerance

33 strategy, lower lethal temperature, rapid cold-hardening, acclimation, deacclimation,

34 phenotypic plasticity, experimental design 


\section{1. Introduction}

36 Temperature constrains the geographic distribution and seasonal activity of insects

37 (Chown and Nicolson, 2004), and therefore can directly or indirectly affect the spread and

38 impact of invasive pests, the success of species introduced for biological control, and the

39 dynamics of native insect populations (Bale and Hayward, 2010). In temperate, polar and

40 montane habitats, the majority of insects spend a large proportion of their life in an

41 overwintering stage, and must survive the low temperatures and accompanying

42 environmental stressors that are associated with winter (Leather et al., 1993; Williams et

43 al., 2015). Similarly, insects in deserts and tropical high mountains can also be regularly

44 exposed to potentially-lethal freezing conditions (Sømme, 1995; Sømme et al., 1996;

45 Sømme and Zachariassen, 1981). Thus, low temperature biology is a key component of

46 insect fitness, and one of the best determinants of insect distribution (Andersen et al.,

47 2015b).

49 Most insects are ectotherms, and as such, their body temperatures are generally

50 similar to the ambient microclimate temperature, and changes in ambient temperature can

51 thus have drastic effects on the physiology of an insect. Thus, measuring low temperature

52 performance (which we refer to loosely here as 'cold tolerance') is an excellent way to

53 incorporate the pervasive effects of temperature in studies ranging from ecological (e.g. van

54 Dooremalen et al., 2013) to molecular (e.g. Reis et al., 2011). In the cold, many insects

55 enter a reversible state of paralysis, called chill coma, at the critical thermal minimum

56 (CT $\left.\mathrm{T}_{\min }\right)$ (MacMillan and Sinclair, 2011a). At sub-zero temperatures, insects risk freezing of 
57 the body fluids, as well as a host of other low temperature injuries (Denlinger and Lee,

58 2010). The ability of an insect to survive at low temperatures is referred to as its cold

59 hardiness, and their responses to low temperature have generally been categorized as chill-

60 susceptible, freeze-avoidant, and freeze-tolerant (Fig. 1, see Section 3) (Bale, 1993).

61

62 There is a long history of the study of insect cold tolerance (Ring and Riegert, 1991;

63 Sømme, 2000), and the sub-discipline has consequently developed its own semantic and

64 methodological traditions. Although there are many excellent reviews on the subject (e.g.

65 Asahina, 1969; Bale, 2002; Block, 1982a; Denlinger and Lee, 2010; Lee, 1991; Salt, 1961;

66 Sinclair et al., 2003b; Zachariassen, 1985), a unified summary of the methods and

67 approaches used in insect cold tolerance is not available. Nevertheless, although care must

68 be taken in the design and interpretation of experiments, measuring insect performance at

69 low temperatures is by no means arcane, and many measurements require no specialist

70 equipment. Our purpose in this review is to explain some of the common measures of

71 insect low temperature biology from a methodological viewpoint, with the intention of

72 making these methods more accessible. We try to identify some of the diverse

73 measurements that are comparable among studies, and our ultimate goal is to reduce some

74 of the trial-and-error inherent in learning a new set of techniques and measurement. Our

75 intended audience is newcomers to the field, students, and (particularly) those who are

76 interested in incorporating low temperature performance into their existing studies, and are

77 looking for an overview of common practices. While there is an unavoidable bias towards

78 the methods or approaches used over the past two decades by the first author, we have tried

79 to encompass alternative approaches wherever possible, and also provide (hopefully) lucid 
80 explanations of why we favour one approach over others. In some cases, we give examples

81 of equipment that has been used in these studies, but we do not intentionally endorse any

82 manufacturer or model.

83

\section{2. Technical and apparatus considerations}

85 Studying insect low temperature performance requires some form of temperature control

86 and measurement. We conclude this section by discussing how to identify and measure an

87 insect's supercooling point (SCP), as measuring the SCP is fundamental to many other

88 measures of insect cold tolerance and provides a useful application of measuring an insect's

89 body temperature during cold exposure.

912.1 Temperature control

92 The simplest cold exposures involve constant low temperatures, and the equipment

93 needed for these exposures is often readily available. For example, an ice-water slurry is at

94 a constant (and precise) $0{ }^{\circ} \mathrm{C}$, many laboratory and domestic refrigerators and cold rooms

95 are held at approximately $+4{ }^{\circ} \mathrm{C}$, and domestic freezers are usually somewhere between -12

96 and $-20^{\circ} \mathrm{C}$. Similarly, refrigerated incubators or refrigerated baths can be easily set to a

97 single temperature. Ultra-low freezers (usually set somewhere between -70 and $-90{ }^{\circ} \mathrm{C}$ ),

98 dry ice $\left(-80^{\circ} \mathrm{C}\right)$, a dry ice-acetone slurry $\left(-78^{\circ} \mathrm{C}\right)$ and liquid nitrogen $\left(-196^{\circ} \mathrm{C}\right)$ all provide

99 constant low temperatures with reasonable precision. Although these latter temperatures

100 are of limited biological relevance when studying living insects, they can be useful if

101 extreme rapid cooling is required. 
103 More commonly, insects are cooled using specialised cooling equipment, such as rate-

104 controlled incubators (e.g. Ju et al., 2011) or refrigerated circulators (e.g. Marshall and

105 Sinclair, 2011). These are often programmable, allowing precise cooling, hold ('soak’ in

106 engineering terminology), and warming programmes. Refrigerated circulators will require

107 a bath liquid (e.g. glycol, alcohol, or a synthetic or mineral oil) for sub-freezing use. In our

108 laboratory we use ethylene glycol (usually mixed 1:1 with water) for moderate low

109 temperatures $\left(\mathrm{c} .-20^{\circ} \mathrm{C}\right.$ ) because it has a lower viscosity than the less-toxic propylene

110 glycol and can be heated. We use methanol (either undiluted or mixed 1:1 with water) for

111 lower temperatures. Samples (in containers) can either be placed directly in the bath, or the

112 batch liquid can be circulated to a cooling stage; in our laboratory we circulate liquid to an

113 insulated aluminium block that is milled with holes to accommodate various sample

114 container sizes, from $1.5 \mathrm{~mL}$ microcentrifuge tubes to $35 \mathrm{~mL}$ standard Drosophila vials.

116 Thermoelectric cooling via Peltier devices can be used to construct efficient cooling

117 devices (especially if the cooling modules are stacked and liquid-cooled), and we have used

118 these in the past (e.g. Sinclair et al., 2003a; Sinclair and Sjursen, 2001); unfortunately,

119 neither of these devices (nor designs for them) are commercially available, so they must be

120 custom-designed and -built (Wharton and Rowland, 1984). However, they are light and

121 robust and use little power, so are ideal for field situations. Another field alternative is to

122 use an endothermic reaction to provide cooling. For example, Sømme et al. (1993) used a

$123 \mathrm{CaCl}_{2} \cdot 6 \mathrm{H}_{2} \mathrm{O} /$ crushed ice mixture to determine SCPs of mites at a field camp in Antarctica.

124 This has the disadvantage of producing chemical waste, but greatly reduces the electricity 
125 requirements for cooling in the field. Another approach to control cooling rate is to use a

126 set-temperature incubator or freezer, and to moderate the cooling rate (if not the final

127 temperature) using insulation, such as a Styrofoam box (e.g. Hao and Kang, 2004).

129 Different methods of cold exposure will have different levels of among-sample and time-

130 related variance. For example, there can be spatial variation among holes in the aluminium

131 block mentioned above, and also within incubators. Thermal fluctuations in incubators

132 (especially at temperatures where compressors may be starting and stopping) can be quite

133 substantial. It is important to view these fluctuations in the context of biological relevance

$134-$ for example, fluctuations of $1{ }^{\circ} \mathrm{C}$ may be unimportant if among-treatment differences are

135 in the order of tens of degrees, but if differences among treatments are subtle (or

136 fluctuations are large), the noise may overpower the signal. Immersing samples in a liquid

137 medium can be used successfully to significantly dampen fluctuations in incubators (e.g.

138 MacMillan et al., 2009). In addition, the temperature actually experienced by an insect may

139 differ from that to which the instrument has been set. Thus, good practice is to always

140 measure the temperature which an animal or group of animals experiences, and to use that

141 as the treatment temperature in analyses.

143 Investigations of insect cold tolerance initially adopted a uniform cooling rate of 144 approximately $1{ }^{\circ} \mathrm{C} / \mathrm{min}$ to standardize results (Salt, 1966). Although this rate is

145 convenient, it is significantly faster than the cooling rates experienced in nature [e.g.

146 Sinclair (2001) measured a maximum cooling rate of $0.01^{\circ} \mathrm{C} / \mathrm{min}$ in alpine microhabitats 
147 in New Zealand -100-fold slower than used in these laboratory studies] and several studies

148 have shown that fast cooling rates lead to additional cold shock (e.g. Bale et al., 1989;

149 Cloudsley-Thompson, 1973; Miller, 1978). In particular, directly plunging an insect into an

150 extreme temperature can significantly decrease survivorship, compared to that achieved

151 during a ramping regime (Nguyen et al., 2014). Conversely, slow cooling rates can make

152 experiments impractically long, and may allow insects to mount a physiological response to

153 cold, such as that observed during rapid cold-hardening (Kelty and Lee, 1999; Nguyen et

154 al., 2014). Most authors currently choose cooling rates that compromise between

155 ecological relevance and time investment; generally 0.1 to $0.5^{\circ} \mathrm{C} / \mathrm{min}$ (e.g. Boardman et

156 al., 2012; Crosthwaite et al., 2011; Renault et al., 2004; Sformo et al., 2011). It is clear that

157 the rate of temperature change does affect the response of the insect (this has also been

158 vigorously discussed in the context of high temperature tolerances, e.g. Santos et al., 2011;

159 Terblanche et al., 2007; Terblanche et al., 2011). Thus, it is important to maintain

160 consistent cooling rates among sites or species for comparisons, and to be cognizant of the

161 assumptions inherent in comparing thermal limits among studies that used different

162 exposure conditions.

1642.2 Temperature measurement

165 Thermocouples are the most common way to measure insect temperatures during

166 cooling. Thermocouples are thin wires that measure temperature via the changes in the

167 voltage output of a bimetallic junction. Usually one thermocouple is used per insect,

168 although it is also possible to increase throughput by carefully placing more than one

169 (small) insect on a thermocouple (e.g. springtails; Sinclair et al., 2006a), or by connecting 
170 multiple thermocouples in series (Nedvěd et al., 1995). Thermocouples differ in the types

171 of metal used, and in the diameter of the wire; any thermocouple type is acceptable if it

172 returns valid measurements over an appropriate range of sub-zero temperatures and is

173 compatible with the recording device. Types K (chromel-alumel) and T (copper-

174 constantan) are most common in insect cold tolerance studies. Smaller-diameter

175 thermocouples will yield a faster response (but are more fragile); we have found 36 AWG

176 thermocouples to be a suitable compromise. Pre-made thermocouples can be purchased in

177 various lengths, or thermocouple wire can be purchased and used to make thermocouples

178 by stripping the wires and twisting them together to create a thermocouple junction

179 (damaged pre-made thermocouples can also be repaired this way). Thermistors can be used

180 in an identical manner to thermocouples for SCP measurements (provided they are small

181 enough), but require specific data acquisition apparatus.

183 To detect freezing, thermocouples must be close enough to (or in contact with) the insect

184 during cooling so they can detect the latent heat of crystallization that indicates ice

185 formation. The lowest temperature that precedes this exotherm is termed the supercooling

186 point (the SCP; Fig. 1). Small insects and eggs can be attached to thermocouples by coating

187 the tip of the thermocouple with a thin film of petroleum jelly, heat sink compound, or

188 vacuum grease, which will adhere to the insect with a light touch (Fig. 2A). This has been

189 particularly successful with springtails and mites (Block, 1982b; Block and Young, 1979).

190 For larger insects (e.g. crickets and beetles), thermocouples may be maintained in contact

191 with the cuticle by placing (or chasing) the insect into a microcentrifuge tube or pipette tip,

192 and packing cotton wool in to hold the animal and a thermocouple in place (Fig. 2B). 
193 Removable adhesive putty (such as the sticky tack used to attach pictures to walls) may

194 also be used to hold the tip of the thermocouple in contact with an insect such as a phasmid

195 (Fig. 2C). Exotherms can often be detected from large organisms even if the thermocouple

196 is not in direct contact with the animal. This approach - placing the thermocouple on the

197 outside of a sealed respirometry chamber containing the animal - has worked for large

198 caterpillars (e.g. Sinclair et al., 2004) and frogs (Sinclair et al., 2013).

200 Data from thermocouples can be acquired directly by a computer using 201 commercially-available thermocouple interfaces, such as the Sable Systems International

202 T2000 (www.sablesys.com) and the Pico Technology TC-08 (www.picotech.com).

203 Alternately, thermocouple data loggers can be used to record data (many will interface 204 directly to a computer). For example, the Hobo UX120 (www.onsetcomp.com), the Grant 205 Squirrel (www.grantinstruments.com), or the Campbell range (www.campbellsci.ca).

Thermocouples can nucleate ice formation, particularly for soft-bodied or moist 208 animals, or if the thermocouple cools more quickly than the insect. For example, Sinclair et 209 al. (2009) found that ice nucleation appeared to begin at the thermocouple attached to 210 drosophilid larvae. Although this impact of thermocouple contact on the SCP is probably 211 negligible for most insects, care should be taken with moist, soft-bodied animals that may 212 be susceptible to inoculative freezing. One way to avoid this problem is to use differential 213 scanning calorimetry (DSC) or infrafred thermography (see below), or to place a very fine 214 (and therefore sensitive) thermocouple near, but not in contact with, the insect. 
216 Differential scanning calorimetry measures heat flow into or out of a sample,

217 usually for chemical analysis. However, the sensitivity and precision of DSC means it can

218 be used to measure freezing in small insects placed in the cooling chamber. Many DSC

219 instruments are cooled by liquid nitrogen, and can therefore be used to measure very low

220 SCPs (e.g. below $\left.-40^{\circ} \mathrm{C}\right)$. Differential Scanning Calorimetry likely avoids artifacts from

221 thermocouple contact, and can be used for multiple small insects at once (e.g. Worland and

222 Convey, 2001). Infrared thermography is another approach to detecting and measuring

223 SCPs without physical contact. This approach has been used successfully to detect SCPs

224 (Palmer et al., 2004), to analyze the ice formation process (Sformo et al., 2009), and to

225 separate freezing of insects from ice formation in their substrate (Koštál et al., 2012). It is

226 now possible to purchase infrared cameras that interface with a smartphone, so the

227 availability of this technology may increase rapidly. Infrared 'thermocouples' are also

228 commercially available, but have not, to our knowledge, been used for measuring insect

229 cold tolerance in the published literature.

$231 \quad 2.3$ Measuring Supercooling Points

232 The supercooling point is the temperature at which freezing begins, and ice

233 formation will usually then proceed from the site of nucleation to other parts of the insect's

234 body (Lee and Lewis, 1985). Many investigations of insect cold tolerance begin with

235 preliminary measurements of the SCP because this provides an anchor point about which

236 the cold tolerance strategy can be determined: an initial step is to determine whether insects

237 survive freezing or not (see Section 3.1). Additionally, changes in the SCP with treatment 
238 or season can indicate biochemical or physiological changes, even if the SCP is not a good

239 measure of survival of low temperatures (Coleman et al., 2014). Because the SCP does not

240 necessarily equate to cold-hardiness, it must be interpreted with caution, and in many cases

241 has limited ecological relevance (Bale, 1987; Baust and Rojas, 1985; Renault et al., 2002).

242 However, for freeze tolerant and freeze-avoidant insects (see below), the SCP does

243 represent an important physiological threshold or lower lethal limit, respectively, and can

244 indicate ecologically-relevant variation in cold tolerance in the latter (e.g. Crosthwaite et

245 al., 2011; van der Merwe et al., 1997; Worland et al., 2006).

Measuring the SCP is as simple as placing an insect in contact with a thermocouple,

248 and cooling it at a reproducible rate to a temperature at which all of the individuals have

249 frozen. The exotherm released by the latent heat of crystallisation is easily detected (Fig.

250 3). The SCP is defined as the lowest temperature recorded prior to the initiation of the

251 exotherm, the duration and shape of which will depend on the size of the insect. Larger

252 insects (e.g. crickets and caterpillars) have more water, and the freezing process takes

253 longer than in small insects (Drosophila and springtails) yielding distinctly different shapes

254 (Fig. 3). In terms of data acquisition, higher sampling rates may be required to capture the

255 SCP of small insects (e.g. springtails) with exotherms that occur rapidly releasing a small

256 amount of heat. In general, a sampling rate of $<1 \mathrm{~Hz}$ is suitable for larger insects, and (from

257 experience) $10 \mathrm{~Hz}$ should be sufficient for even small insects, insect eggs, Collembola, and

258 mites. If the cooling method results in fluctuations in temperature, or there is a large

259 amount of condensation present, it is sometimes possible to detect artifactual freezing 
260 events that are not the result of the insect freezing (Fig. 3). A simple solution to this is to

261 include a thermocouple without an insect attached, to allow interpretation of any artifacts.

263 Supercooling points are usually measured on 20-30 individuals, which provides a

264 robust sample size with which to assess the shape of the distribution of SCPs. Although

265 cold-hardy populations of insects often have unimodal SCP distributions, some species

266 (and also microarthropods like springtails and mites) have bimodal SCP distributions

267 (Cannon and Block, 1988; Sinclair et al., 2006a; Figure 4). Bimodal SCP distributions can

268 arise due to variation in the presence of ice nucleators or differences in body composition.

269 For example, springtails that have recently fed (introducing ice nucleators into the gut

270 leading to a high group of SCPs; Sømme, 1982), and/or moulted (removing nucleators;

271 Worland et al., 2006) have very different SCPs. Starved adult Alphitobius diaperinus

272 beetles also had a bimodal SCP distribution, but this was due to s starvation $\times$ sex

273 interaction such that the SCPs of males decreased with starvation, but the SCps of females

274 did not (Salin et al., 2000). Although techniques do exist to determine breakpoints in

275 bimodal distributions (e.g. Aldrich, 1987), many authors simply decide on an a priori high

276 group-low group divider by collating all SCPs and visually assessing a histogram for an

277 obvious break and then compare the frequency, means or medians of the high and low

278 groups among treatments using standard methods (e.g. Sinclair et al., 2006a). Because

279 supercooling point distributions are seldom normal, and abnormally high or low values

280 from one or two individuals can skew the entire distribution, care must be taken in using

281 parametric statistics when comparing SCPs among samples or treatments. In addition,

282 because larger insects contain more water, they have a higher probability of freezing, and 
283 therefore higher SCPs. One approach to account for this potential confounding factor is to 284 incorporate body size (e.g. as mass) into statistical model (or conduct analyses on residuals 285 of a regression of body size on SCP), demonstrated by Hahn et al. (2008). This may be 286 necessary if (e.g.) dietary treatments yield individuals of markedly different body sizes. An 287 alternate approach (when possible) is to use broadly size-matched individuals for 288 comparison.

Ice formation can take only a few seconds in small animals, particularly at low

291 SCPs. By contrast, freezing can be a slow process in larger insects. For example, the peak 292 of the exotherm in 3-5 g alpine weta, Hemideina maori (Orthoptera: Anostostomatidae) 293 occurs approximately $1.5 \mathrm{~min}$ after the SCP, but at that point only $5 \%$ of the body water is 294 converted into ice. Ice content reached an equilibrium 70-80 \% after c. 8 h (Figure 5; 295 Ramløv and Westh, 1993). Because the amount of heat released is proportional to the 296 quantity of water converted to ice, it is tempting to use the exotherm area to estimate ice 297 content. However, a thermocouple does not capture the heat release accurately enough to 298 quantify ice formation from its measurements (or to usefully interpret the highest 299 temperature the thermocouple records), so the amount of ice formed must be measured 300 calorimetrically (e.g. Block et al., 1998; Layne and Blakeley, 2002; Ramløv and Westh, 301 1993). 


\section{Cold tolerance strategy}

304 An insect's cold tolerance strategy describes how it survives temperatures where its

305 body fluids might be expected to freeze. Chill susceptible species are killed by cold in the

306 absence of internal ice formation (Bale, 1993). Chill susceptibility is sometimes referred to

307 as chilling- or cold-intolerance. For example, the larvae of the false codling moth,

308 Thaumatotobia leucotreta (Lepidoptera: Tortricidae), freeze between $-13^{\circ} \mathrm{C}$ and $-22^{\circ} \mathrm{C}$ but

309 are killed by brief exposures between $-8{ }^{\circ} \mathrm{C}$ and $-12{ }^{\circ} \mathrm{C}$ (Boardman et al., 2012). Chill

310 susceptibility is not a strategy per se, but represents the impact of cold on a majority of

311 insects, particularly those that are not at all cold-hardy, and including many insects from

312 the tropics or in temperate areas during the growing season. Freeze avoidance (sometimes

313 referred to as freezing-intolerance) refers to species that survive cold in the absence of

314 internal ice formation, but that are killed by any internal ice. Most of these insects

315 maintain their body fluids in a supercooled state (i.e. remain liquid below the melting

316 point) via accumulation of cryoprotectants and prevention of ice nucleation (Salt, 1961).

317 For example, prepupae of the emerald ash borer, Agrilus planipennis can survive prolonged

318 exposures to subzero temperatures, provided they do not freeze, and in the winter have

319 SCPs below $-25^{\circ} \mathrm{C}$ (Crosthwaite et al., 2011). Cryoprotective dehydration is a special case

320 of freeze avoidance, whereby ice formation is prevented via removal of freezable water to

321 environmental ice through a permeable cuticle. This dehydrates the animal, increasing the

322 concentration of solutes to an equilibrium point that is unfreezeable at a given temperature

323 (Holmstrup et al., 2002). For example, larvae of the Antarctic midge, Belgica antarctica

324 (Diptera: Chironomidae), overwinter in the soil matrix, which dehydrates them to the point

325 that they cannot freeze (Elnitsky et al., 2008). Finally, Freeze-tolerant insects tolerate the

326 formation of ice in their body tissues and fluids. For example, the pre-pupae of the golden 
327 rod gall fly Eurosta solidaginis (Diptera: Tephritidae) freeze around $-8{ }^{\circ} \mathrm{C}$ but can survive

328 with more than $60 \%$ of their body converted to ice, with mortality beginning below $-25{ }^{\circ} \mathrm{C}$

329 (Morrissey and Baust, 1976). Many temperate species switch from one strategy to another

330 at the onset of winter (Salt, 1961), and the presence or absence of external ice can also

331 determine cold tolerance strategy (e.g. Shimada and Riihimaa, 1988).

333 Defining a species' cold tolerance strategy is essential for interpreting the SCP, and

334 for determining the subsequent approach to measuring lethal temperatures (see Section 4).

335 However, while the cold tolerance strategy helps explain how insects respond to and

336 survive exposure to low temperatures, it does not predict or quantify survival in a particular

337 winter or overwintering site, nor does it designate absolute levels of cold toelrance (Bale,

338 1993; Sinclair, 1999). For example, in temperate southwestern Ontario, Canada, winter

339 temperatures regularly drop below $-25^{\circ} \mathrm{C}$, yet freeze avoidant (e.g. Crosthwaite et al.,

340 2011), freeze-tolerant (e.g. Marshall and Sinclair, 2011) and chill-susceptible (Udaka and

341 Sinclair, 2014) species are all abundant. Thus, cold tolerance strategy is not a substitute for

342 understanding lethal limits, and cannot be used on its own to predict survival. Despite its

343 inability to predict cold tolerance, the cold tolerance strategy can indicate potential

344 prediction and control methods (see Leather et al., 1993, for a comprehensive review). For

345 example, freeze-avoidant species may be susceptible to conditions that promote ice

346 formation, such as the application of ice nucleators (e.g. Fields, 1993; Lee et al., 1994). 


\subsection{Determining cold tolerance strategy}

349 Cold tolerance strategy is best inferred from two pieces of information gathered

350 from the same individual during a cold exposure: 1) Whether or not the insect froze, and 2)

351 whether or not it survived (Table 1; Salt, 1961). Cold tolerance strategy can be determined

352 with a small number of individuals cooled, in contact with thermocouples, to a temperature

353 close to the SCP. By rewarming all of the individuals once half of them have frozen

354 (evident from exotherms), it is possible to generate a population of insects that includes

355 individuals that did and did not freeze, but that were all exposed to the same low

356 temperature. The cold tolerance strategy can then be determined from the survival of these

357 individuals (Tables 1 and 2; see section 4.1 for a discussion of survival metrics).

Many studies (e.g. Bemani et al., 2012; Slabber and Chown, 2004) derive cold

360 tolerance strategy from separate measurements of SCP and lower lethal temperature

361 (reasoning that $\mathrm{LT}_{50}<\mathrm{SCP}=$ freeze tolerant; $\mathrm{LT}_{50}=\mathrm{SCP}=$ freeze avoidant; and $\mathrm{LT}_{50}>\mathrm{SCP}=$

362 chill susceptible). While this indirect method is suitable if there are very large differences

363 between SCP and LLT [e.g. Drosophila melanogaster dies at approximately $-5{ }^{\circ} \mathrm{C}$, but has

364 an SCP below $-15{ }^{\circ} \mathrm{C}$ (Czajka and Lee, 1990)], it is imprecise if SCP and LLT are in close

365 proximity. In particular, it can be difficult to distinguish between freeze avoidance and

366 chill-susceptibility if the latter insects are cold-hardy.

Ice formation is a slow process, particularly in larger insects (Fig. 5). Thus,

369 survival of brief exposures below the SCP may not reflect survival of 'complete' freezing

370 (i.e. freezing to an equilibrium ice content). While it is possible to measure the ice content 
371 of insects (e.g. Block et al., 1998; Layne and Blakeley, 2002; Ramløv and Westh, 1993),

372 holding insects below the SCP for a longer period (e.g. overnight) can indicate whether the

373 animal is tolerant of equilibrium ice content (Dennis et al., 2015; Sinclair et al., 1999).

374 Insects that do not survive complete ice formation [termed 'partially freeze tolerant' by

375 Sinclair (1999)] may nevertheless use freeze tolerance as a component of their cold

376 tolerance strategy, depending on the environmental conditions, particularly if sub-freezing

377 conditions are brief (e.g. Sømme et al., 1996; Sømme and Zachariassen, 1981), although

378 this has not been well-explored. Voituron et al. (2002) hypothesised that this partial freeze

379 tolerance may be an important intermediate step in the evolution of freeze tolerance.

380 Similarly, although some insects may survive short periods of acute cold exposure if they

381 remain unfrozen, freeze-avoidant insects must remain unfrozen when supercooled for long

382 periods (Sømme, 1996). The ability to remain supercooled is easily tested by cooling

383 insects to close to the SCP, and holding them at that temperature, for example, overnight

384 (e.g. Crosthwaite et al., 2011).

385

$386 \quad$ Some species of insect tolerate freezing only if ice formation is initiated

387 (inoculated) at relatively high subzero temperatures (Lee, 2010). For example, larvae of

388 Chymomyza costata,(Diptera: Drosophilidae) were initially classified as freeze-avoidant,

389 but if freezing is inoculated by contact with ice crystals, then diapausing larvae will survive

390 below $-80^{\circ} \mathrm{C}$ (Shimada and Riihimaa, 1988). This is most relevant to species expected to

391 overwinter in moist conditions, and ice formation can be inoculated by placing an animal in

392 contact with a moist substrate (e.g. Boardman et al., 2012; Koštál and Havelka, 2000),

393 applying ice (e.g. Layne et al., 1990), or applying a known ice nucleator, such as silver 
394 iodide (e.g. Strachan et al., 2011). Cold tolerance strategy can then be assessed as 395 described above.

397 To identify cryoprotective dehydration, insects need to be exposed to low 398 temperatures in the presence of (but not actually in contact with) ice. In the field, this 399 occurs in frozen soil or moss (Holmstrup, 2014), and the most common laboratory method 400 is to confine insects in a mesh or perforated container within a larger sealed container that 401 contains crushed ice (e.g. Elnitsky et al., 2008; Sørensen and Holmstrup, 2011; Udaka and 402 Sinclair, 2014), which is exposed to cold, usually in an incubator or other large chamber. 403 The hallmarks of cryoprotective dehydration are a decrease in water content (usually 404 measured gravimetrically), such that the concentration of solutes in the body fluids 405 increases to yield a melting point approximately equivalent to the ambient temperature, 406 rendering the insect essentially unfreezable [Fig. 6; see Holmstrup (2014) for an extensive

407 review]. Simultaneously, the insect may also accumulate low molecular weight 408 cryo/anydroprotectants, such as trehalose. Thus, insects using cryoprotective dehydration 409 will have both reduced water content and depressed supercooling points, and these should 410 decrease in concert with decreasing temperature.

413 their winter-relevant cold-tolerance strategy after appropriate seasonal cues. For example, 414 Eurosta solidaginis is chill-susceptible in the summer, but freeze-tolerant in the winter (Lee 415 and Hankison, 2003), and Pyrrhocoris apterus (Hemiptera: Pyrrhocoridae) is chill- 
416 susceptible in the summer, but freeze-avoidant in the winter (Koštál and Simek, 2000).

417 Second, there may be variation in cold tolerance strategy among populations, for example

418 Pieris rapae (Lepidoptera Pieridae) is freeze-avoidant in northern Europe, but freeze

419 tolerant in central Siberia ( $\mathrm{Li}$ and Zachariassen, 2007). Third, some species may switch

420 strategy entirely. For example, overwintering larvae of the beetle Dendroides canadensis,

421 (Coleoptera: Pyrochroidae) switched from freeze tolerant in the winter of 1978-1979 to

422 freeze avoidant in 1981-1982 (Horwath and Duman, 1984). We will discuss approaches to

423 elicit this plasticity in Section 6.

424

\section{4. Lower lethal limits}

426 Lower lethal limits quantify the temperatures that kill an insect population or species

427 under specified conditions. Lethal temperatures are usually expressed as a proportional

428 lethal temperature $\left(\mathrm{LT}_{\mathrm{x}}\right)$, the point where $\mathrm{x} \%$ of exposed individuals die. For example, the

$429 \mathrm{LT}_{50}$ is the median lethal temperature, expected to kill $50 \%$ of a population. The Lower

430 Lethal Temperature (LLT) is the temperature at which all individuals are killed (the $\mathrm{LT}_{100}$;

431 functionally, this is often expressed as the highest temperature at which measured survival

432 was zero). The lowest temperature at which no individuals are killed has been called the

433 Upper Limit of the Cold Injury Zone (ULCIZ; Nedvěd, 1998). Note that any assessment of

434 the lower lethal temperature is dependent also on the exposure time (see section 4.1).

436 The LLT estimates an absolute limit to low temperature survival and, as such, can

437 provide information about likelihood of survival under a given set of conditions (e.g. 
438 Hatherly et al., 2005), and allow survival to be compared under circumstances where the

439 SCP is uninformative about survival (e.g. for chill-susceptible or freeze-tolerant species;

440 Baust and Rojas, 1985). Other thresholds, such as the $\mathrm{LT}_{90}$, indicate thresholds associated

441 with specified levels of probability, and are therefore useful for assessing the risk of

442 mortality or survival under a given set of conditions. Used in conjunction with the

443 supercooling point, lower lethal temperatures can be used to indirectly estimate cold

444 tolerance strategy (see Section 3.1). However, estimates of LLT are sensitive to exposure

445 conditions, especially cooling and rewarming rates. For example, survival of Chymomyza

446 costata larvae exposed to $-40{ }^{\circ} \mathrm{C}$ decreases from $>90 \%$ to zero when the cooling rate is

447 increased from 0.1 to $1{ }^{\circ} \mathrm{C} / \mathrm{min}$ (Shimada and Riihimaa, 1988). Thus, if the laboratory

448 conditions do not reflect the salient features of the conditions in nature, laboratory-derived

449 LLT estimates may over- or under-estimate the probability of mortality in the field.

$451 \quad 4.1$ Estimating Lower lethal limits

$452 \quad$ Lower lethal limits are usually determined by exposing individual insects (or more

453 commonly, groups of individuals) to a pre-determined range of test temperatures, each for

454 the same fixed period of time. Approximately five temperatures spanning the range of 0 to

$455100 \%$ mortality are usually sufficient to determine the $\mathrm{LT}_{50}$, although additional

456 temperatures are sometimes added to obtain a finer-scale estimate of $\mathrm{LT}_{50}$. After cold

457 exposure, insects are returned to recovery conditions (e.g. room temperature or the rearing

458 temperature), and allowed to recover before survival is assessed. 
461 nature, and convenience. Lower lethal temperature estimates that examine responses to

462 short cold exposures (1-2 h; e.g. Clarke et al., 2013) estimate survival of an acute, transient

463 extreme temperature. In many habitats, these might reflect the minimum temperature

464 experienced over a winter. However, a case can be made for longer exposures, such as

465 overnight treatments, that may be more ecologically-relevant (Sømme, 1996). Ideally these

466 measurements (or assessment of the risk of survival) will be interpreted in the context of

467 known overwintering or exposure conditions, such as temperature records from

468 microclimate monitoring (e.g. Udaka and Sinclair, 2014). A similar approach can be used

469 to measure high temperature tolerance (ULT) and lethal time at a low temperature (LLt).

470 The latter is particularly relevant for risk assessment for insects that may be exposed to

471 prolonged cold during refrigerated shipping (e.g. Beaudry et al., 1998) or while

472 overwintering under snow (Pauli et al., 2013), or to extensive periods below freezing

473 (Sømme, 1996). To estimate LLt, a similar approach to LLT is taken, but with exposures to

474 a set temperature across a range of pre-determined times. Ultimately, LLT and LLt are both

475 important information: for most species, thermal tolerance is a temperature-time surface

476 (see Nedvěd, 1998; Nedvěd et al., 1998), which has seldom been fully parameterized.

478 Determining LLT is, by necessity, an iterative process. Ideally, survival metrics, 479 SCP, and cold tolerance strategy have already been determined, and can help to determine a 480 starting point, with the goal of anchoring the analysis with exposures to temperatures that 481 yield $0 \%$ and $100 \%$ survival, with additional replicates to fill the intervening space (e.g. 482 Udaka and Sinclair, 2014). An alternative approach is to expose groups of insects to a pre- 
483 determined set of temperatures (e.g. at $5{ }^{\circ} \mathrm{C}$ intervals; Bouchard et al., 2006; Kleynhans et

484 al., 2014). This approach has advantages in design and execution; in particular, it can allow

485 random assignment of insects to treatments, allow all of the exposures to be made on a

486 single day, and is more suitable for using longer-term measures of survival.

Assessing mortality after cold exposures requires a consistent (and ecologically-

489 relevant) estimate of whether or not an insect is alive, and an appropriate time period to 490 capture mortality caused by cold, but exclude mortality from other causes. Ideally, survival

491 to reproduction indicates that an insect has retained fitness after cold exposure (Baust and 492 Rojas, 1985), but this is surprisingly rare as a metric. Survival to the reproductive stage for 493 immature insects (i.e. successful eclosion as an adult) is an ecologically-relevant estimate 494 of mortality that is more common (e.g. Strachan et al., 2011; Williams et al., 2014).

495 However, for species that do poorly in captivity, those with long life cycles, or because of 496 pressure to obtain data, most researchers choose simple metrics of survival that can be 497 rapidly assessed. These metrics are often a coordinated movement (standing or a righting 498 response, e.g. MacMillan and Sinclair, 2011b; Nyamukondiwa et al., 2011; Tomcala et al., 499 2006), although resumption of feeding, or a more 'athletic' ability (running, jumping, or 500 flying) would also be appropriate. More simple measures can be problematic, as they can 501 lack an assessment of biologically-relevant performance. For example, cold-exposed 502 insects can be injured and able to move in an uncoordinated fashion, but unable to perform 503 more complex functions. Such individuals have been characterized as 'injured' (Koštál et 504 al., 2006; MacMillan and Sinclair, 2011b) or 'moribund' (Sinclair, 1997). These 
505 individuals are usually considered 'dead' in analyses on the basis that since they could not 506 reproduce, their fitness is zero.

508 Mortality is usually assessed within the first 24 hours to a few days after cold exposure.

509 Thus, lethal temperature estimates may ignore mortality that occurs in the longer term, and 510 also disregard sub-lethal effects on behavior or reproductive capacity (Baust and Rojas,

511 1985). For example, adults of the freeze-tolerant carabid Pterostichus brevicornis will

512 survive for a few days when frozen and thawed under sub-optimal conditions, but die after

513 a week (Miller, 1969). By contrast, it is sometimes possible to significantly reduce the

514 recovery period needed for a survival assessment. For example, Udaka \& Sinclair (2014)

515 found no difference in survival estimates of cold-exposed Curculio glandium (Coleoptera:

516 Curculionidae) larvae made $15 \mathrm{~min}$ and $24 \mathrm{~h}$ after rewarming. Choosing a timeframe and

517 metric for assessment of mortality will thus depend on the goal of the study; when

518 assessing potential for overwintering survival, longer-term survival and reproductive-

519 capacity measures may be most appropriate.

520

$521 \quad$ Lower lethal temperature experiments are usually conducted with sequential

522 exposure of different groups of individuals to a range of temperatures (e.g. Bouchard et al.,

523 2006; Figure 7). The response variable is percent or proportion mortality (or survival, for

524 the optimistic). Thus, the unit of replication in an LLT study is a group of individuals, not

525 the individuals themselves; five groups of ten individuals gives $n=5$, whereas one group of

526 fifty (the same number of animals) gives $n=1$. Because survival is expressed as a 
527 proportion, groups of fewer than five are less useful than larger groups, but the balance

528 between resolution and replication usually leads most researchers to use groups of 5-10.

529 There are statistical power advantages to treating each individual's survival separately

530 (using, for example, survival or failure time analyses, e.g. Jakobs et al., 2015), however,

531 researchers rarely take this approach, perhaps because most exposures are conducted in

532 groups. Even if individuals are individually housed, a single exposure treatment should

533 probably be considered a group, to avoid pseudoreplication (McArdle, 1996). When using

534 multiple experimental groups of individuals (e.g. 3-5) at each temperature, 3-5 independent

535 LLT curves do not need to be generated; instead, the order of exposure temperatures can be

536 randomized to yield replication, and all the data considered in the same model. Because

537 lethal limits are estimated using a regression approach, it is not necessary to expose insects

538 to precisely determined temperatures; it is sufficient to have an accurate measurement of

539 the temperature to which each group was exposed.

540

541 Lethal temperatures can be estimated graphically by plotting the survival

542 proportions by temperature and 'eyeballing' the lethal limits (Fig. 7A). However, a formal

543 statistical analysis of survival is relatively simple, and can yield values for $\mathrm{LT}_{50}$ and LLT

544 (and any other level of mortality), and also variance or confidence intervals around those

545 estimates. Because survival has a binomial error distribution (individuals are either alive or

546 dead), linear models, such as ANOVA, are inappropriate for determining LLT (although

547 ANOVA may be suitable for comparing among models, particularly in R, where this is a

548 common approach - e.g. Crawley, 2005). A logistic regression (Fig. 7B) with logit link and

549 binomial error distribution (i.e. a probit analysis) is, a priori, most appropriate (e.g. Bürgi 
550 and Mills, 2010; Sinclair, 1997). Alternative forms of survival analysis (e.g. Ransberry et

551 al., 2011) are inappropriate for LLT analyses, because they assume that an individual is

552 followed throughout an exposure until it dies - clearly this is not the case when the

553 individual has to be rewarmed to ascertain survival.

554

555 5. Chill Coma

556 When cooled, a majority of insects will slow down and eventually lose the ability to

557 move. At a specific temperature, an insect will cross a threshold (the critical thermal

558 minimum, $\mathrm{CT}_{\min }$ ), into a reversible state of paralysis, known as chill coma. (Hazell and

559 Bale, 2011). Because insects cannot move, feed, reproduce, or evade predators while in

560 chill coma, the $\mathrm{CT}_{\min }$ provides a useful lower limit to insect function (Andersen et al.,

561 2015b; David et al., 1998; Gibert et al., 2001; MacMillan and Sinclair, 2011a), and is often

562 used to approximate insect cold tolerance because it is broadly correlated to lethal limits

563 (Andersen et al., 2015b). Resumption of movement after rewarming (chill coma recovery,

564 CCR) is usually measured as the amount of time it takes insects to recover from a

565 standardised period in chill coma (David et al., 1998; Gibert et al., 2001; MacMillan and

566 Sinclair, 2011a). Additionally, because chill coma is usually non-lethal, it is possible to use

567 insects for further experiments, or even breed from them, allowing for selection

568 experiments (e.g. Bertoli et al., 2010; Franke et al., 2012; Mori and Kimura, 2008; Telonis-

569 Scott et al., 2009).

571 Although insects may accumulate injuries (or die) while in chill coma (MacMillan 
572 and Sinclair, 2011a), $\mathrm{CT}_{\min }$ and CCR are not measures of cold tolerance, but rather of

573 resistance to the effects of cold (although it provides a useful estimate of thermal biology

574 for comparative purposes; Andersen et al., 2015b). $\mathrm{CT}_{\min }$ is also limited as a measurement

575 of resistance to cold, because it can only be measured in mobile life stages; specifically,

576 monitoring when an insect enters chill coma requires identifying and timing the loss or

577 recovery of some form of movement. Some insects become immobile when disturbed (e.g.

578 some species of stick insects and beetles), making it difficult to determine the $\mathrm{CT}_{\text {min }}$.

579 Finally, not all species have a $\mathrm{CT}_{\min }$. For example, freezing occurs before chill coma in the 580 freeze-avoidant Antarctic springtail, Isotoma klovstadi (Sinclair et al., 2006a) and in some 581 freeze-tolerant cockroaches (Sinclair and Chown, 2005).

$583 \quad 5.1$ Chill coma Onset $\left(C T_{\min }\right)$

$584 \quad$ The $\mathrm{CT}_{\min }$ is the most common measure of the threshold at which chill coma onset

585 (CCO) occurs. A similar measure of maintenance of performance at low temperatures,

586 knockdown time, could be used to determine the amount of time at a predetermined

587 temperature after which coordination is lost (cf. Hoffmann et al., 1997). However, it is

588 unclear whether these two approaches measure the same physiological phenomenon

589 (MacMillan and Sinclair, 2011a), so $\mathrm{CT}_{\min }$ and knockdown time should not be used

590 interchangeably. Because most literature currently uses $\mathrm{CT}_{\min }$ we suggest that this provides

591 the measure of chill coma onset that is most readily comparable among studies (and is the 592 approach we will discuss further below), assuming assessments have been made under 593 comparable conditions. 
595 To measure the $\mathrm{CT}_{\text {min }}$, the point at which an insect loses its ability to move needs to

596 be identified. This could be through direct observation during cooling (e.g. Klok and

597 Chown, 1997), or through identification of failure to remain in a chamber, or on a perch

598 (e.g. Huey et al., 1992). These observations need to be coupled to a measurement of

599 temperature in the chamber (or even at the surface of the insect). When direct observations

600 are being made, an ideal response will be repeatable among researchers and individual

601 animals, involuntary, and easily discerned, although stimulus is often necessary at low

602 temperatures because insects move more slowly (MacMillan and Sinclair, 2011a). Motor

603 responses include the righting response (standing after being placed on back or knocked

604 over; e.g. David et al., 1998), response to a stimulus such as prodding with a probe (e.g.

605 Klok and Chown, 1997), or coordinated standing (e.g. Koštál et al., 2004); an appropriate

606 species-specific response can be identified via a pilot study. We note (from experience) that

607 some species are simply not tractable for $\mathrm{CT}_{\text {min }}$ studies; for example, stick insects are

608 immobile when threatened - this is it hard to tell if a stick insect is not moving because it

609 cannot move, or because it will not move.

610

611 The cooling rate used for $\mathrm{CT}_{\min }$ measurement is a balance between being slow enough to

612 ensure that the insect's body temperature does not lag behind the chamber (leading to

613 inaccurate estimation of $\mathrm{CT}_{\min }$ ), and being fast enough to avoid substantial physiological

614 (e.g. acclimation) responses during cooling (Huey et al., 1992). Thus, most $\mathrm{CT}_{\min }$ protocols

615 require less than $2 \mathrm{~h}$ to complete the entire assay. Most studies use cooling rates between

6160.1 and $0.25^{\circ} \mathrm{C} / \mathrm{min}$ for determining $\mathrm{CT}_{\min }$ (e.g. Kleynhans et al., 2014; MacMillan and

617 Sinclair, 2011b; Terblanche et al., 2006). Note that there has been considerable recent 
618 debate on the 'correct' rate for measuring high temperature tolerances (Rezende et al., 619 2011; Santos et al., 2011; Terblanche et al., 2007; Terblanche et al., 2011), and it is

620 therefore imperative to clearly report the conditions when describing $\mathrm{CT}_{\min }$ experiments. To

621 expedite measurements, it should be possible to rapidly reduce the temperature from the

622 rearing temperature to an intermediate temperature (e.g. $10^{\circ} \mathrm{C}$ ), allowing equilibration

623 before cooling at the pre-determined rate. To our knowledge, the consequences of different 624 equilibration times in such protocols have not been explored.

625

626 A variety of methods can be used to cool individual insects for manual $\mathrm{CT}_{\min }$ 627 determination, including controlled-temperature incubators (e.g. Hu and Appel, 2004), 628 immersing insects in tubes or containers in the bath of a refrigerated circulator (e.g. Klok 629 and Chown, 1997), or using purpose-built chambers cooled by fluid circulated from 630 refrigerated circulators (e.g. MacMillan and Sinclair, 2011b; Sinclair et al., 2006b). Higher 631 throughput has been achieved by placing a population of insects into a column with 632 baffles/perches (Huey et al., 1992; Ransberry et al., 2011; Renault and Lalouette, 2012).

633 The insects lose coordination at the $\mathrm{CT}_{\min }$ as the column is cooled, and can be collected at 634 intervals (or counted electronically; e.g. Shuman et al., 1996) as they fall out the bottom of 635 the column, with each interval representing individuals that entered chill coma over a 636 specific time period.

637

638 Other methods to observe the $\mathrm{CT}_{\min }$ have also been explored. Renault et al. (1999)

639 used a microbalance to detect activity during declining temperature to identify the $\mathrm{CT}_{\min }$ in

640 a beetle. Similarly, Hazell et al. (2008) describe making a video recording as an insect is 
641 cooled, and then extracting from the video the point at which the last voluntary movement

642 was made [Everatt et al. (2013) describe a means to automate this measurement].

643 Alternately, physiological measurements may be plausibly developed that allows the $\mathrm{CT}_{\min }$

644 to be identified from a physiological threshold. Such a measure could use heart function

645 (Andersen et al., 2015a), metabolic rate (Lighton and Turner, 2004; Sinclair et al., 2004), or

646 automated activity monitoring (MacMillan et al., 2012a), although most of these

647 approaches are low-throughput.

648

649 Individual- or group-based studies of insect $\mathrm{CT}_{\min }$ usually use 10-20 individuals per

650 treatment (e.g. Clarke et al., 2013; Jumbam et al., 2008; Klok and Chown, 1997; Renault

651 and Lalouette, 2012), but the knockdown column approach allows for larger sample sizes

652 (as many as 263 flies in Ransberry et al., 2011). Critical thermal minima can be compared

653 among treatments by treating each individual as a replicate, and using conventional

654 statistics (e.g. ANOVA; Klok and Chown, 1997), or non-linear approaches such as

655 Generalized Linear Models, if not normally distributed. Critical thermal minima may also

656 satisfy the assumptions of survival analyses such as Accelerated Failure Time models (e.g.

657 Ransberry et al., 2011).

658

$659 \quad 5.2$ Chill Coma Recovery

660 Because chill coma is reversible and movement is easy to detect in mobile insects,

661 recovery from chill coma is also used as a measure of cold tolerance. Although some

662 authors have measured this as a temperature at which movement reinitiates (e.g. Sinclair et

663 al., 2006a), current research suggests that chill coma recovery is a complex interplay 
664 between the rate of loss of homeostasis, the low temperature equilibrium attained, and the

665 rate and threshold for recovery (Fig. 8). Thus, the most-used metric tends to be time until

666 recovery under standardised conditions (Chill Coma Recovery time; CCRT), since this

667 minimises confounding variables (e.g. confounding the rate of recovery versus a

668 temperature threshold for recovery). Here we will therefore discuss only CCRT, although

669 we note that although it is simple to measure under standardised conditions, it is difficult to

670 interpret in ecological context.

671

672 To measure CCRT, insects are exposed to a temperature-time combination that

673 induces chill coma, and returned to a recovery temperature (often the rather vague 'room

674 temperature' - we recommend at least reporting and measuring that temperature). The

675 insects are observed, and the time taken to resume a pre-determined behavior or activity is

676 recorded. Movements and behaviors include coordinated leg movement (Halle et al.,

677 2015), standing or walking (e.g. Macdonald et al., 2004), righting response, or abdominal

678 contractions (e.g. Macmillan et al., 2012b). It should be possible to use video analysis to

679 automate CCRT (e.g. by modifying the method described by Hazell et al., 2008 for $\mathrm{CT}_{\min }$ ).

680 Insects can be cold-exposed via refrigerated baths or incubators (see Section 2.1); however,

681 because many insects enter chill coma around $0{ }^{\circ} \mathrm{C}$, an ice-water slurry is often sufficient

682 (e.g. Gibert et al., 2001; Nilson et al., 2006; Ransberry et al., 2011). Recovery can be

683 observed in any clear container; a convenient approach for smaller insects, such as

684 Drosophila is to transfer the insect to 6- or 12-well cell culture plates, which allows

685 multiple individuals to be observed simultaneously. The recovery temperature will likely

686 influence the CCRT, however, this has not been well-explored (Macmillan et al., 2012b). 
689 of a working day), and yield chill coma recovery times within a convenient range (we 690 suggest 20-30 minutes). It is important that the resolution of observation is compatible

691 with the expected variation in CCR. For example, the difference between 15 and 20

692 minutes (a $33 \%$ increase) is easily distinguished, but the difference between 3 and 4

693 minutes may not be if each individual is observed at $30 \mathrm{~s}$ intervals. There is often a bi-

694 phasic relationship between exposure time (or temperature) and CCRT (David et al., 2003;

695 Macdonald et al., 2004; Macmillan et al., 2012b), and in the plateau region (which may be

696 narrow or broad), the loss of ion and water balance is putatively at an equilibrium

697 (MacMillan and Sinclair, 2011a; Macmillan et al., 2012b); this ensures that differences

698 among individuals, treatments or populations are primarily due to variation in the recovery

699 processes (Fig. 8). Thus, comparing CCR across a combination of temperatures and times

700 is a useful first step to ensure that the exposure conditions used to induce chill coma result

701 in a recovery time that is convenient, appropriate for distinguishing between groups, and

702 within the plateau region.

703

704 Because of its simplicity, sample sizes for CCRT can be larger than those for $\mathrm{CT}_{\text {min, }}$, 705 typically 10-20 individuals/treatment (e.g. Andersen et al., 2015b; David et al., 2003;

706 Findsen et al., 2013; Gibert et al., 2001; Macdonald et al., 2004; Ransberry et al., 2011).

707 Because only a limited number of individuals can be observed at a time (typically $<20$, if

708 the observation interval is $20-30 \mathrm{~s}$ ), we recommend that the treatments in each observation

709 set be randomised. Analysis approaches mirror those of $\mathrm{CT}_{\min }$, although because the CCRT 
710 has a lower bound (an insect can't take a negative time to recover), survival analyses or

711 generalized linear models might be more appropriate than linear parametric statistics such

712 as ANOVA. Chill coma recovery time is often compared among treatments measured

713 together as a way to examine relative differences; however, because of the diversity of

714 exposure temperatures and times (Table 3), recovery metric and recovery temperatures, we

715 recommend caution when comparing CCRT among species, studies, and laboratories.

716

717 6. Detecting variation in low temperature performance among

718 individuals, populations, seasons and treatments

719 Phenotypic plasticity is the ability of an organism with a given genotype to express

720 different phenotypes. Phenotypic plasticity in thermal biology includes hardening,

721 acclimation, which occurs in the laboratory, and acclimatization, which happens in nature,

722 although the mechanisms of acclimation and acclimatization are assumed to be similar

723 (Kingsolver, 2009; Tattersall et al., 2012), but different to the mechanisms underlying

724 hardening (Sinclair and Roberts, 2005; Teets and Denlinger, 2013). Developmental

725 plasticity is the response to conditions experienced during development (e.g. Sisodia and

726 Singh, 2010), while maternal (or trans-generational) effects refer to epigenetic signals

727 passed from the mother to offspring that determine phenotype (e.g. Magiafoglou and

728 Hoffmann, 2003). The time scale of phenotypic plasticity responses can range from

729 minutes or hours (often termed 'hardening' responses) to long-term shifts, such as seasonal

730 changes in cold tolerance (Fischer and Karl, 2010; Sinclair and Roberts, 2005). Genetic

731 adaptation is plasticity on an evolutionary scale, and may play out among populations or

732 species (Gibert and Huey, 2001; Kellermann et al., 2009; Sinclair et al., 2012). 
734 All of these levels of variation in physiology can dramatically alter cold tolerance;

735 thus, one way to account for this variation is to attempt to induce as much plasticity in cold

736 tolerance as possible. Although this approach may not reveal the absolute limits of cold

737 tolerance (and cannot determine the adaptive significance of the plasticity), it can at least

738 indicate the presence of plasticity, and put a study into the context of a species’ plasticity.

739 Here we define and explain the major sources of plasticity in insect low temperature

740 performance, and discuss approaches that can be used to detect this plasticity.

741

$742 \quad 6.1$ Phenotypic plasticity of low temperature biology in nature

743 Phenotypic plasticity of cold tolerance is readily observed in populations of insects

744 in nature at multiple timescales, most commonly as seasonal changes in cold tolerance. For

745 example, field-collected larvae of the freeze-avoidant codling moth Cydia pomonella

746 (Lepidoptera: Torticidae) are more cold-tolerant during the winter than in the summer

747 (Khani and Moharramipour, 2010). Similarly, emerald ash borer have depressed

748 supercooling points in winter (Crosthwaite et al., 2011), and freeze-tolerant alpine

749 cockroaches have a lower $\mathrm{LT}_{50}$ in winter (Sinclair, 1997), both of which enhance

$750 \quad$ overwintering survival.

751 In addition to seasonal variation in cold tolerance, there is also evidence of

752 phenotypic plasticity over shorter timescales in nature. For example, survival of cold

753 exposure increases at night and decreases during the day in field-caged Drosophila

754 melanogaster (Overgaard and Sørensen, 2008). Similarly, the supercooling point

755 distribution of field-collected Antarctic springtails Desoria klovstadi (Collembola: 
756 Isotomidae) decreases markedly between midday and midnight, reflecting changes in

757 ambient temperature (Sinclair et al., 2003c). Thus, it is important to consider these natural

758 variations in cold tolerance when determining the overwintering potential of an insect.

$760 \quad 6.2$ Rapid cold-hardening

761 Rapid cold-hardening (RCH) is the shortest time-scale of plastic responses, and

762 describes enhanced survival of temperature extremes after a brief (minutes to c. $3 \mathrm{~h}$ ) pre-

763 exposure to sub-lethal temperatures (reviewed by Teets and Denlinger, 2013). Rapid cold

764 hardening can affect a range of cold tolerance parameters; for example, by decreasing LLT

765 (Lee et al., 1987), $\mathrm{CT}_{\min }$ and CCRT (Ransberry et al., 2011), and even modifying the SCP in

766 some cases (e.g. Worland and Convey, 2001). Thus, RCH rapidly shifts insects from a non-

767 cold-hardy to a cold-hardy state in life stages that are not normally cold-tolerant, or at times

768 of year when seasonal cold tolerance has not yet been acquired. Because of this rapid shift

769 in cold tolerance, parameters like LLT may be substantially underestimated if RCH is not

770 taken into account. For example, non-diapausing adults of the elm leaf beetle

771 Xanthogaleruca luteola (Coleoptera: Chrysomelidae), have only $15 \%$ survival after $1 \mathrm{~h}$ at

$772-7^{\circ} \mathrm{C}$, but survival increases to $90 \%$ if the $-7^{\circ} \mathrm{C}$ exposure is preceded by $4 \mathrm{~h}$ at $0{ }^{\circ} \mathrm{C}$ (Lee

773 et al., 1987). Rapid cold-hardening is mainly expressed in chill-susceptible species, but has

774 been reported in freeze-tolerant and freeze-avoidant species as well (Lee and Denlinger, 775 2010).

776

777 RCH may be induced by slow cooling, diurnal cycles and mild low temperatures

778 (usually -2 to $+4{ }^{\circ} \mathrm{C}$ ) (Teets and Denlinger, 2013). Unlike the heat shock response (see 
779 Harrison et al., 2012), RCH does not normally require a recovery period after the pre-

780 exposure to express the enhanced cold tolerance. Nevertheless, including a recovery period

781 is commonly incorporated in laboratory assessments of RCH (e.g. Jakobs et al., 2015;

782 Nunamaker, 1993; Sinclair and Chown, 2003). Because the mechanisms of RCH are still

783 poorly understood (Teets and Denlinger, 2013), it is not entirely clear whether these

784 different treatments eliciting 'RCH’ are actually triggering the same physiological

785 response, so it is important to report the methods fully, and potentially include several

786 different treatments in a study (e.g. Nunamaker, 1993).

$788 \quad 6.3$ Acclimation, deacclimation and acclimatization

789 Cold acclimation and acclimatization are processes that improve cold-hardiness

790 over a span of days-to-weeks, within a single life stage. Acclimation can have a marked

791 effect on cold tolerance; for example, a six-week cold acclimation increased survival of the

792 rusty grain beetle, Cryptolestes ferrugineus, from 1.4 days to 24 days at $-10{ }^{\circ} \mathrm{C}$. Cold-

793 acclimation regimes may be based on measured field conditions (e.g. Jakobs et al., 2015),

794 or based on convenience (e.g. the $4{ }^{\circ} \mathrm{C}$ of many domestic and laboratory refrigerators).

795 Notably, acclimation under fluctuating temperatures usually leads to greater cold tolerance

796 (Colinet et al., 2015). Although laboratory acclimation can never properly replicate field

797 conditions, the aim of incorporating laboratory acclimation is to provide some indication of

798 the extent of plasticity, and therefore the extent to which potential cold tolerance is being

799 underestimated.

800

801 Both acclimation and acclimatization are reversible; in the C. ferrugineus example 
802 above, the enhanced cold tolerance was lost within a week of returning the insects to the 803 rearing temperature of $30^{\circ} \mathrm{C}$ (Fields et al., 1998), a process termed deacclimation. In the 804 field, deacclimation could occur during seasonal temperature variation (e.g. mid-winter 805 thaws). For example, Sobek-Swant et al. (2012) found that winter-acclimatized freeze806 avoidant emerald ash borer prepupae had increased SCPs and decreased glycerol 807 concentrations after exposure to mid-winter warm spells $\left(+10\right.$ and $\left.+15^{\circ} \mathrm{C}\right)$, and that this 808 deacclimation was not reversed when the prepupae were returned to $-10{ }^{\circ} \mathrm{C}$. Deacclimation 809 is rarely included in cold tolerance studies, but is clearly relevant when attempting to 810 understand the plasticity of cold tolerance, particularly in relation to variable environments.

$812 \quad 6.4$ Developmental and cross-generational plasticity

813 Developmental plasticity is the non-reversible change in phenotype of one life stage

814 that is decided by the experience of an earlier life stage (for review and examples, see

815 Davidowitz and Nijhout, 2004; Kingsolver and Huey, 2008; Nylin and Gotthard, 1998).

816 For example, Bicyclus butterflies reared at a high temperature have slower chill coma

817 recovery than those reared at a lower temperature, regardless of their adult experience

818 (Franke et al., 2012); by contrast, flour beetles raised under high temperatures have faster

819 chill coma recovery than their low temperature counterparts (Scharf et al., 2015).

820 Developmental plasticity is thus important when considering overwintering, because entry

821 into appropriate physiological state for overwintering may be initiated during an earlier life 822 stage.

824 In some species, the overwintering phenotype is determined not by an individual's 
825 experience, but by the experience of the previous generation. For example, diapause by

826 Aedes eggs is determined by the photoperiod experienced by the mother (Denlinger and

827 Armbruster, 2014). Both developmental plasticity and trans-generational effects are easily

828 missed in laboratory studies, and could therefore mean that laboratory studies

829 underestimate low temperature performance in the field, but, to our knowledge, have been

830 rarely explored.

831

8326.5 Detecting phenotypic plasticity

833 The complexity of phenotypic plasticity means that it cannot be completely

834 encompassed in simple laboratory experiments. Thus, we emphasise that the goal of

835 laboratory studies of phenotypic plasticity is to provide an indication of the extent to which

836 the full cold tolerance potential is being captured by the laboratory experiments. A

837 valuable alternative or complement to these experiments is to conduct seasonal

838 measurements of the cold tolerance of field-collected (or at least field-caged) individuals

839 (e.g. Baust and Miller, 1970; Crosthwaite et al., 2011; Koštál et al., 2014; Udaka and

840 Sinclair, 2014). These field studies may provide the best, and most ecologically-relevant,

841 estimate of the cold tolerance changes driven by the combined effects of temperature,

842 photoperiod and trans-generational and developmental plasticity.

844 The general approach to detecting plasticity of cold tolerance in the laboratory is to

845 apply pre-treatments or acclimation treatments, and measure some (preferably ecologically-

846 relevant) response variable or phenotype, such as $\mathrm{CT}_{\text {min, }}$ SCP or LLT. The treatments

847 could include both brief pre-treatments (to elicit rapid cold-hardening) and longer 
848 treatments (to elicit acclimation responses). One convenient approach to detecting

849 plasticity phenotypes is the 'discriminating temperature' approach (Powell and Bale, 2004).

850 In this approach, a discriminating temperature (one that causes $\sim 80 \%$ mortality) is chosen

851 from preliminary investigations. Insects are then exposed to various pre-treatments and

852 acclimations, and their survival is measured only at that temperature. While the

853 discriminating temperature approach does not estimate the change in absolute limits of

854 survival, increased survival does provide evidence of plasticity, which can be explored in

855 greater detail as required. Discriminating temperatures are most often used in studies

856 documenting the RCH response (e.g. Sinclair and Chown, 2006), but it can also be used to

857 explore other sources of plasticity, including heat shock and acclimation (e.g. Rajamohan

858 and Sinclair, 2008, 2009). However, SCP is a relevant metric for freeze-avoidant species

859 (e.g. Worland and Convey, 2001), and $\mathrm{CT}_{\min }$ or CCRT may also be used to identify

860 plasticity in a relatively high-throughput fashion (e.g. Everatt et al., 2013; Fischer et al.,

861 2010; Hoffmann et al., 2005; Sisodia and Singh, 2010).

862

863 There is an almost infinite variety of combinations of potential cues for phenotypic

864 plasticity (Table 4), so if the goal is simply to detect plasticity, it is most convenient to

865 choose a small combination of treatments that encompass a variety of cues and time

866 frames. Because it is the plasticity itself, not the precise cues, that is important, it can be

867 useful to deliberately conflate photoperiod and temperature cues to maximize the likelihood

868 of inducing plasticity. For example, acclimation treatments often combine short day length

869 with low temperatures, without controlling for each factor independently (e.g. Jakobs et al.,

870 2015). 
872 We suggest four basic treatment approaches that encompass the main components

873 of plasticity (Fig. 9): A) Some form of pre-treatment intended to elicit rapid cold-hardening

874 (Lee et al., 1987); B) A 5-10 day low temperature-short day length treatment to elicit an

875 acclimation response (e.g. Slabber and Chown, 2005); C) Development at low temperature

876 with a short day length (e.g. Colinet and Hoffmann, 2012); and D) Fluctuating temperatures

877 and decreasing photoperiod, for example based upon climate (e.g. a nearby weather station)

878 or microclimate (e.g. Jakobs et al., 2015). The precise temperature and photoperiod details,

879 as well as the duration of the treatments, will depend on the natural history and timing of

880 the life cycle of the organism, its lifespan, and its propensity for laboratory culture. A

881 developmental acclimation treatment will not necessarily be possible for a univoltine

882 species, or one that is not readily reared in the laboratory. Deacclimation has been poorly

883 explored, but is usually achieved through exposures to warmer-than-usual winter

884 temperatures for a period of days (see Section 6.2).

886 Using the discriminating temperature approach means that a simple comparison of

887 low temperature survival by each treatment to that of the control can reveal significant

888 effects of the plasticity treatments. In this approach, the unit of replication is the group (see

889 also section 4.1), and 5-10 groups per treatment should provide adequate power. As with

890 LLT measurements, it is best if replicates are randomized, and controls run alongside

891 treatment groups. Some care may need to be taken to control for aging if this is a potential

892 factor in lengthy acclimation treatments, as aging can decrease (e.g. Halle et al., 2015) or

893 increase (e.g. Lalouette et al., 2010) cold tolerance, depending on the species. Survival of 
894 pre-treated groups can be directly compared to that of controls, using t-tests, ANOVA with 895 planned comparisons, or GLZ (with binomial error distributions) and planned comparisons.

896 If ANOVA or t-tests are used, note that the binomial error distribution inherent in survival

897 data is inappropriate for these tests, but an arcsine-square-root transformation can

898 normalize data somewhat prior to analysis.

900 Interpreting data from the discriminating temperature approach is difficult, because 901 it allows the detection of plasticity, but does not quantify the magnitude of that plasticity. If 902 survival increases markedly (e.g. from 20 to 100\%), it makes it clear that LLT has been 903 underestimated, but to obtain parametric predictions it will be best to construct new LLT 904 curves with individuals in which plasticity has been detected (see section 4.1). Note that it 905 is possible that plasticity treatments may also modify the cold tolerance strategy (e.g. 906 Shimada and Riihimaa, 1988), thereby modifying the approach to determining potential for 907 overwintering survival.

909 7. Suggested workflow

910 Leaving aside the thrill of basic discovery, there are two main reasons to measure

911 low temperature performance in a previously-uninvestigated insect: 1) to provide a detailed 912 description of its cold tolerance, perhaps in the context of an invasion or interesting habitat

913 or 2) to provide a measure of low temperature performance to facilitate comparisons among 914 treatments or populations, or to round out a study incorporating a wider array of stressors.

915 In the latter case, the priority is therefore to choose a metric that is both biologically- 
916 relevant and easy to measure, while in the former case, the emphasis may be on

917 determining thresholds that can be explored in the context of the habitat. No single

918 measure will be appropriate for all species or situations, and (like all work on living

919 organisms), it is best to begin with some basic observations. In this final section, we

920 provide two suggested approaches, one for a general exploration of low temperature

921 biology, the other for choosing a measure of low temperature performance.

922

923 To begin a comprehensive description of insect cold tolerance, we recommend

924 beginning with two simple observations: SCP (Section 2.3) and $\mathrm{CT}_{\min }$ (Section 5.1). These

925 two parameters provide anchor points for designing experiments to further investigate

926 CCRT, cold tolerance strategy, and lethal limits (Fig. 10). In turn, these measures help to

927 determine appropriate response variables for examining phenotypic plasticity (Section 6).

928 Examples of this approach can be found in many of the papers cited in this review (e.g.

929 Koštál et al., 2014; Sinclair and Chown, 2005; Slabber and Chown, 2004). Subsequent

930 investigations of the biochemistry underlying plasticity and cold tolerance, for example,

931 changes in hemolymph composition, can then be incorporated into suitably-informed

932 sampling and analyses, that are beyond the scope of this review (see, e.g., Bale and

933 Hayward, 2010; Duman et al., 1991; Lee, 2010; Storey and Storey, 1991, 2013;

934 Zachariassen, 1991; Zachariassen and Kristiansen, 2000; Zachariassen et al., 2004). As we

935 have discussed, the biology of the organism will determine the utility of metrics used -

936 overwintering and diapausing insects, for example, often do not merit measurement of

$937 \mathrm{CT}_{\min }$, so $\mathrm{CT}_{\min }$ is consequently absent from studies on many overwintering insects (e.g.

938 Koštál et al., 2014). A reasonable description of cold tolerance can be made with small 
939 sample size, by carefully combining individual measures of SCP, survival, and lower lethal

940 temperature (e.g. Sinclair and Chown, 2005).

941

942 Choosing a single metric as a proxy for cold tolerance in a wider study can be more

943 difficult. If a more-or-less complete description of cold tolerance is available, it is a simple

944 matter to compare (or correlate) the possible variables, and choose a metric that is broadly

945 representative of thermal tolerances and logistically convenient for the organism at hand.

946 In Drosophila, for example, the $\mathrm{CT}_{\min }$ provides a reasonably good proxy for cold tolerance

947 (including $\mathrm{LT}_{50}$ ), and is thus a parsimonious choice for comparison among treatments

948 (Andersen et al., 2015b). If this information is not available, then choosing an appropriate

949 metric is going to be based partially on the life history of the organism, and striking a

950 useful measure may be somewhat dependent on luck. In Table 5, we present the

951 advantages and disadvantages of using each metric to facilitate choosing a particular one to

952 suit the aim of the study.

953

\section{8. Final remarks}

955 Low temperatures are one of the key limiting factors for the distribution and

956 performance of many terrestrial insects, and cold is a key component of overwintering

957 stress in temperate, polar, and alpine habitats. Because of this, the signal-to-noise ratio for

958 measures of cold tolerance is large, providing useful information in comparing insects at

959 multiple scales in time and space. In particular, to fully understand the implications of

960 climate change, there is increasing need to expand beyond growing-season studies to 
961 biology in the fall and winter (Gallinat et al., 2015; Williams et al., 2015), for which low

962 temperature biology is a critical component. Thus, there is significant incentive for insect

963 ecologists, both basic and applied, to measure some aspect of low temperature biology. It

964 is our hope that this review will be of some value in demystifying what are, for the most

965 part, relatively straightforward methods; our advice is, of course, our own, and we hope

966 that we have also provided enough reference to the literature to allow a newcomer to the

967 field to reflect on, and judge for themselves, our recommendations.

968

969 We end with the observation that the field of insect cold tolerance research has

970 always been small and diverse. However, our experience has been that it is a welcoming

971 community, and that for many of its members, cold tolerance is something of a side-

972 interest. Nevertheless, for many of those researchers, the fascination of insect biology at

973 low temperatures means that while insect cold tolerance is not necessarily the most

974 substantial part of their research portfolio, it is almost always a favourite and we therefore

975 end by encouraging others to join us.

976

977 9. Acknowledgements

978 This document is derived from one originally commissioned by the Canadian Food

979 Inspection Agency under Research Contract number 3872188. Thanks to Martin Damus

980 and Ruth Jakobs for comments on earlier drafts, Stephanie Sobek-Swant for some initial

981 discussions, and Eric Moise, Kennan Oyen, Inon Scharf, and two anonymous reviewers for

982 feedback on a later draft. BJS thanks David Wharton, Bill Block, Roger Worland and Hans 
983 Ramløv for introducing him to the methods of insect cold tolerance. LVF was supported by 984 a postgraduate scholarship from the Natural Sciences and Engineering Research Council of 985 Canada. Work by BJS on insect cold tolerance in Canada has been supported by the 986 Natural Sciences and Engineering Research Council of Canada, the Canadian Food 987 Inspection Agency, The Ontario Ministry for Research and Innovation, the Canadian 988 Foundation for Innovation, The University of Western Ontario, and the National Institutes 989 of Health (USA).

990

991 
993Aldrich, J.C., 1987. Graphical analyses of bimodal frequency distributions with an example 994 using Cancer pagurus. J Zool, Lond 211, 307-319.

995Andersen, J.L., Macmillan, H.A., Overgaard, J., 2015a. Temperate Drosophila preserve cardiac 996 function at low temperature. J Insect Physiol 77, 26-32.

997Andersen, J.L., Manenti, T., Sorensen, J.G., MacMillan, H.A., Loeschcke, V., Overgaard, J., 998 2015b. How to assess Drosophila cold tolerance: chill coma temperature and lower lethal 999 temperature are the best predictors of cold distribution limits. Funct Ecol 29, 55-65.

1000Asahina, E., 1969. Frost resistance in insects. Adv Insect Physiol 6, 1-49.

1001Bale, J.S., 1987. Insect cold hardiness: Freezing and supercooling - an ecophysiological 1002 perspective. J Insect Physiol 33, 899-908.

1003Bale, J.S., 1993. Classes of insect cold hardiness. Funct Ecol 7, 751-753.

1004Bale, J.S., 2002. Insects and low temperatures: from molecular biology to distributions and 1005 abundance. Phil Trans R Soc Lond B 357, 849-861.

1006Bale, J.S., Hansen, T.N., Nishino, M., Baust, J.G., 1989. Effect of cooling rate on the survival of 1007 larvae, pupariation, and adult emergence of the gallfly Eurosta solidaginis. Cryobiol 26, $1008 \quad 285-289$.

1009Bale, J.S., Hayward, S.A.L., 2010. Insect overwintering in a changing climate. J Exp Biol 213, 1010 980-994.

1011Baust, J.G., Miller, L.K., 1970. Seasonal variations in glycerol content and its effect on cold1012 hardiness in the Alaskan carabid beetle, Pterostichus brevicornis. J Insect Physiol 16, 9791013990.

1014Baust, J.G., Rojas, R.R., 1985. Insect cold hardiness: Facts and fancy. J Insect Physiol 31, 7551015759.

1016Beaudry, R.M., Moggia, C.E., Retamales, J.B., Hancock, J.F., 1998. Quality of 'Ivanhoe' and 1017 'Bluecrop' blueberry fruit transported by air and sea from Chile to North America. 1018 Hortscience 33, 313-317.

1019Bemani, M., Izadi, H., Mandian, K., Khani, A., Samih, M.A., 2012. Study on the physiology of 1020 diapause, cold hardiness and supercooling point of overwintering pupae of the pistachio 1021 fruit hull borer, Arimania comaroffi. J Insect Physiol 58, 897-902. 
1022Bertoli, C.I., Scannapieco, A.C., Sambucetti, P., Norry, F.M., 2010. Direct and correlated 1023 responses to chill-coma recovery selection in Drosophila buzzatii. Entomol Exp Appl 134, 1024 154-159.

1025Block, W., 1982a. Cold hardiness in invertebrate poikilotherms. Comp Biochem Physiol 73A, 1026 581-593.

1027Block, W., 1982b. Supercooling points of insects and mites on the Antarctic Peninsula. Ecol 1028 Entomol 7, 1-8.

1029Block, W., Wharton, D.A., Sinclair, B.J., 1998. Cold tolerance of a New Zealand alpine 1030 cockroach, Celatoblatta quinquemaculata (Dictyoptera, Blattidae). Physiol Entomol 23, 110316.

1032Block, W., Young, S.R., 1979. Measurement of supercooling in small arthropods and water 1033 droplets. Cryo-Lett 1, 85-91.

1034Boardman, L., Grout, T.G., Terblanche, J.S., 2012. False codling moth Thaumatotibia leucotreta 1035 (Lepidoptera, Tortricidae) larvae are chill-susceptible. Insect Sci 19, 315-328.

1036Bouchard, R.W., Carrillo, M.A., Kells, S.A., Ferrington, L.C., 2006. Freeze tolerance in larvae of 1037 the winter-active Diamesa mendotae Muttkowski (Diptera : Chironomidae): a contrast to 1038 adult strategy for survival at low temperatures. Hydrobiologia 568, 403-416.

1039Bürgi, L.P., Mills, N.J., 2010. Cold tolerance of the overwintering larval instars of light brown 1040 apple moth Epiphyas postvittana. J Insect Physiol 56, 1645-1650.

1041Burton, V., Mitchell, H.K., Young, P., Petersen, N.S., 1988. Heat-shock protection against cold 1042 stress of Drosophila melanogaster. Mol Cell Biol 8, 3550-3552.

1043Cannon, R.J.C., Block, W., 1988. Cold tolerance of microarthropods. Biol Rev 63, 23-77.

1044Chen, C.-P., Denlinger, D.L., Lee, R.E., Jr, 1987. Cold-shock injury and rapid cold hardening in 1045 the flesh fly Sarcophaga crassipalpis. Physiol Zool 60, 297-304.

1046Chown, S.L., Nicolson, S.W., 2004. Insect Physiological Ecology. Mechanisms and Patterns. 1047 Oxford University Press, Oxford.

1048Clarke, M.W., Thompson, G.J., Sinclair, B.J., 2013. Cold Tolerance of the Eastern Subterranean 1049 Termite, Reticulitermes flavipes (Isoptera: Rhinotermitidae), in Ontario. Environ Entomol $1050 \quad 42,805-810$.

1051Cloudsley-Thompson, J.L., 1973. Factors influencing the supercooling of tropical arthropoda, 1052 especially locusts. J Nat Hist 7, 471-480. 
1053Coello Alvarado, L.E., MacMillan, H.A., Sinclair, B.J., 2015. Chill-tolerant Gryllus crickets

1054 maintain ion balance at low temperatures. J Insect Physiol 77, 15-25.

1055Coleman, P.C., Bale, J.S., Hayward, S.A.L., 2014. Cross-generation plasticity in cold hardiness is

1056 associated with diapause, but not the non-diapause developmental pathway, in the blow fly

1057 Calliphora vicina. J Exp Biol 217, 1454-1461.

1058Colinet, H., Hoffmann, A.A., 2012. Comparing phenotypic effects and molecular correlates of

1059 developmental, gradual and rapid cold acclimation responses in Drosophila melanogaster.

1060 Funct Ecol 26, 84-93.

1061Colinet, H., Sinclair, B.J., Vernon, P., Renault, D., 2015. Insects in fluctuating thermal

1062 environments. Annu Rev Entomol 60, 123-140.

1063Crawley, M.J., 2005. Statistics: an introduction using R. Wiley, Chichester.

1064Crosthwaite, J.C., Sobek, S., Lyons, D.B., Bernards, M.A., Sinclair, B.J., 2011. The

1065 overwintering physiology of the emerald ash borer, Agrilus planipennis Fairmaire

1066 (Coleoptera: Buprestidae). J Insect Physiol 57, 166-173.

1067Czajka, M.C., Lee, R.E., Jr., 1990. A rapid cold-hardening response protecting against cold shock 1068 injury in Drosophila melanogaster. J Exp Biol 148, 245-254.

1069David, J.R., Gibert, P., Moreteau, B., Gilchrist, G.W., Huey, R.B., 2003. The fly that came in

1070 from the cold: geographic variation of recovery time from low-temperature exposure in

1071 Drosophila subobscura. Funct Ecol 17, 425-430.

1072David, R.J., Gibert, P., Pla, E., Petavy, G., Karan, D., Moreteau, B., 1998. Cold stress tolerance

1073 in Drosophila: Analysis of chill coma recovery in D. melanogaster. J Therm Biol 23, 291-

1074299.

1075Davidowitz, G., Nijhout, H.F., 2004. The physiological basis of reaction norms: The interaction

1076 among growth rate, the duration of growth and body size. Integr Comp Biol 44, 443-449.

1077Denlinger, D.L., Armbruster, P.A., 2014. Mosquito Diapause. Annu Rev Entomol 59, 73-93.

1078Denlinger, D.L., Lee, R.E., 2010. Insect Low Temperature Biology. Cambridge University Press,

1079 Cambridge.

1080Dennis, A.B., Dunning, L.T., Sinclair, B.J., Buckley, T.R., 2015. Parallel molecular routes to cold 1081 adaptation in eight genera of New Zealand stick insects. Sci Reports 5, 13965.

1082Duman, J.G., Wu, D.W., Xu, L., Tursman, D., Olsen, T.M., 1991. Adaptations of insects to

1083 subzero temperatures. Q Rev Biol 66, 387-410. 
1084Elnitsky, M.A., Hayward, S.A.L., Rinehart, J.P., Denlinger, D.L., Lee, R.E., 2008.

1085 Cryoprotective dehydration and the resistance to inoculative freezing in the Antarctic 1086 midge, Belgica antarctica. J Exp Biol 211, 524-530.

1087Everatt, M.J., Bale, J.S., Convey, P., Worland, M.R., Hayward, S.A.L., 2013. The effect of 1088 acclimation temperature on thermal activity thresholds in polar terrestrial invertebrates. J 1089 Insect Physiol 59, 1057-1064.

1090Fields, P.G., 1993. Reduction of cold tolerance of stored-product insects by ice-nucleating-active 1091 bacteria. Environ Entomol 22, 470-476.

1092Fields, P.G., Fleurat-Lessard, F., Lavenseau, L., Febvay, G., Peypelut, L., Bonnot, G., 1998. The 1093 effect of cold acclimation and deacclimation on cold tolerance, trehalose and free amino 1094 acid levels in Sitophilus granarius and Cryptolestes ferrugineus (Coleoptera). J Insect 1095 Physiol 44, 955-965.

1096Findsen, A., Andersen, J.L., Calderon, S., Overgaard, J., 2013. Rapid cold hardening improves 1097 recovery of ion homeostasis and chill coma recovery time in the migratory locust, Locusta 1098 migratoria. J Exp Biol 216, 1630-1637.

1099Fischer, K., Dierks, A., Franke, K., Geister, T.L., Liszka, M., Winter, S., Pflicke, C., 2010.

1100 Environmental Effects on Temperature Stress Resistance in the Tropical Butterfly Bicyclus 1101 anynana. PLoS ONE 5, e15284.

1102Fischer, K., Karl, I., 2010. Exploring plastic and genetic responses to temperature variation using 1103 copper butterflies. Clim Res 43, 17-30.

1104Franke, K., Dierks, A., Fischer, K., 2012. Directional selection on cold tolerance does not 1105 constrain plastic capacity in a butterfly. BMC Evol Biol 12, 14.

1106Gallinat, A.S., Primack, R.B., Wagner, D.L., 2015. Autumn, the neglected season in climate 1107 change research. Trends Ecol Evol 30, 169-176.

1108Gibert, P., Huey, R.B., 2001. Chill-coma temperature in Drosophila: Effects of developmental 1109 temperature, latitude, and phylogeny. Physiol Biochem Zool 74, 429-434.

1110Gibert, P., Moreteau, B., Petavy, G., Karan, D., David, J.R., 2001. Chill-coma tolerance, a major 1111 climatic adaptation among Drosophila species. Evolution 55, 1063-1068.

1112Hahn, D.A., Martin, A.R., Porter, S.D., 2008. Body Size, but Not Cooling Rate, Affects

1113 Supercooling Points in the Red Imported Fire Ant, Solenopsis invicta. Environ Entomol

$1114 \quad 37,1074-1080$. 
1115Halle, S., Nowizki, A., Scharf, I., 2015. The consequences of parental age for development, body 1116 mass and resistance to stress in the red flour beetle. Biol J Linn Soc 115, 305-314. 1117Hao, S.G., Kang, L., 2004. Supercooling capacity and cold hardiness of the eggs of the 1118 grasshopper Chorthippus fallax (Orthoptera : Acrididae). Eur J Entomol 101, 231-236. 1119Harrison, J.F., Woods, H.A., Roberts, S.P., 2012. Ecological and Environmental Physiology of 1120 Insects. Oxford, New York.

1121Hatherly, I.S., Bale, J.S., Walters, K.F.A., 2005. UK winter egg survival in the field and 1122 laboratory diapause of Typhlodromips montdorensis. Physiol Entomol 30, 87-91. 1123Hazell, S.P., Bale, J.S., 2011. Low temperature thresholds: Are chill coma and CT(min) 1124 synonymous? J Insect Physiol 57, 1085-1089.

1125Hazell, S.P., Pedersen, B.P., Worland, M.R., Blackburn, T.M., Bale, J.S., 2008. A method for the 1126 rapid measurement of thermal tolerance traits in studies of small insects. Physiol Entomol 1127 33, 389-394.

1128Hoffmann, A.A., Dagher, H., Hercus, M., Berrigan, D., 1997. Comparing different measures of 1129 heat resistance in selected lines of Drosophila melanogaster. J Insect Physiol 43, 393-405. 1130Hoffmann, A.A., Shirriffs, J., Scott, M., 2005. Relative importance of plastic vs genetic factors in 1131 adaptive differentiation: geographical variation for stress resistance in Drosophila 1132 melanogaster from eastern Australia. Funct Ecol 19, 222-227.

1133Holmstrup, M., 2014. The ins and outs of water dynamics in cold tolerant soil invertebrates. $\mathrm{J}$ 1134 Therm Biol 45, 117-123.

1135Holmstrup, M., Bayley, M., Ramløv, H., 2002. Supercool or dehydrate? An experimental 1136 analysis of overwintering strategies in small permeable Arctic invertebrates. Proc Natl 1137 Acad Sci USA 99, 5716-5720.

1138Horwath, K.L., Duman, J.G., 1984. Yearly variations in the overwintering mechanisms of the 1139 cold-hardy beetle Dendroides canadensis. Physiol Zool 57, 40-45.

$1140 \mathrm{Hu}$, X.P., Appel, A.G., 2004. Seasonal variation of critical thermal limits and temperature 1141 tolerance in Formosan and eastern subterranean termites (Isoptera : Rhinotermitidae). Env 1142 Entomol 33, 197-205.

1143Huey, R.B., Crill, W.D., Kingsolver, J.G., Weber, K.E., 1992. A Method for Rapid Measurement 1144 of Heat or Cold Resistance of Small Insects. Funct Ecol 6, 489-494. 
1145Jakobs, R., Gariepy, T.D., Sinclair, B.J., 2015. Adult plasticity of cold tolerance in a continental1146 temperate population of Drosophila suzukii. J Insect Physiol 79, 1-9.

1147Ju, R.T., Xiao, Y.Y., Li, B., 2011. Rapid cold hardening increases cold and chilling tolerances 1148 more than acclimation in the adults of the sycamore lace bug, Corythucha ciliata (Say) 1149 (Hemiptera: Tingidae). J Insect Physiol 57, 1577-1582.

1150Jumbam, K.R., Jackson, S., Terblanche, J.S., McGeoch, M.A., Chown, S.L., 2008. Acclimation 1151 effects on critical and lethal thermal limits of workers of the Argentine ant, Linepithema 1152 humile. J Insect Physiol 54, 1008-1014.

1153Kellermann, V., van Heerwaarden, B., Sgro, C.M., Hoffmann, A.A., 2009. Fundamental 1154 Evolutionary Limits in Ecological Traits Drive Drosophila Species Distributions. Science $1155325,1244-1246$.

1156Kelty, J.D., Lee, R.E., Jr., 1999. Induction of rapid cold hardening by ecologically relevant 1157 cooling rates in Drosophila melanogaster. J Insect Physiol 45, 719-726.

1158Khani, A., Moharramipour, S., 2010. Cold hardiness and supercooling capacity in the 1159 overwintering larvae of the codling moth, Cydia pomonella. J Insect Sci 10, 12. 1160Kingsolver, J.G., 2009. The Well-Temperatured Biologist. Amer Nat 174, 755-768. 1161Kingsolver, J.G., Huey, R.B., 2008. Size, temperature, and fitness: three rules. Evol Ecol Res 10, 1162 251-268.

1163Kleynhans, E., Conlong, D.E., Terblanche, J.S., 2014. Host plant-related variation in thermal 1164 tolerance of Eldana saccharina. Entomol Exp Appl 150, 113-122.

1165Klok, C.J., Chown, S.L., 1997. Critical thermal limits, temperature tolerance and water balance 1166 of a sub-Antarctic caterpillar, Pringleophaga marioni (Lepidoptera: Tineidae). J Insect 1167 Physiol 43, 685-694.

1168Koštál, V., Havelka, J., 2000. Diapausing larvae of the midge Aphidoletes aphidimyza (Diptera:

1169 Cecidomyiidae) survive at subzero temperatures in a supercooled state but tolerate 1170 freezing if inoculated by external ice. Eur J Entomol 96, 433-436.

1171Koštál, V., Korbelova, J., Rozsypal, J., Zahradnickova, H., Cimlova, J., Tomcala, A., Simek, P., 1172 2011a. Long-Term Cold Acclimation Extends Survival Time at 0 degrees $\mathrm{C}$ and Modifies 1173 the Metabolomic Profiles of the Larvae of the Fruit Fly Drosophila melanogaster. PLoS 1174 One 6, 10. 
1175Koštál, V., Miklas, B., Dolezal, P., Rozsypal, J., Zahradnickova, H., 2014. Physiology of cold 1176 tolerance in the bark beetle, Pityogenes chalcographus and its overwintering in spruce 1177 stands. J Insect Physiol 63, 62-70.

1178Koštál, V., Simek, P., 2000. Overwintering strategy in Pyrrhocoris apterus (Heteroptera): the 1179 relations between life-cycle, chill tolerance and physiological adjustments. J Insect 1180 Physiol 46, 1321-1329.

1181Koštál, V., Simek, P., Zahradnickova, H., Cimlova, J., Stetina, T., 2012. Conversion of the chill 1182 susceptible fruit fly larva (Drosophila melanogaster) to a freeze tolerant organism. Proc $1183 \quad$ Natl Acad Sci U S A 109, 3270-3274.

1184Koštál, V., Vambera, J., Bastl, J., 2004. On the nature of pre-freeze mortality in insects: water 1185 balance, ion homeostasis and energy charge in the adults of Pyrrhocoris apterus. J Exp 1186 Biol 207, 1509-1521.

1187Koštál, V., Yanagimoto, M., Bastl, J., 2006. Chilling-injury and disturbance of ion homeostasis in 1188 the coxal muscle of the tropical cockroach (Nauphoeta cinerea). Comp Biochem Physiol 1189 B 143, 171-179.

1190Koštál, V., Zahradnickova, H., Simek, P., 2011b. Hyperprolinemic larvae of the drosophilid fly, 1191 Chymomyza costata, survive cryopreservation in liquid nitrogen. Proc Natl Acad Sci USA 1192 108, 13041-13046.

1193Lalouette, L., Vernon, P., Amat, H., Renault, D., 2010. Ageing and thermal performance in the 1194 sub-Antarctic wingless fly Anatalanta aptera (Diptera: Sphaeroceridae): older is better. 1195 Biol Lett 6, 346-349.

1196Layne, J.R., Blakeley, D.L., 2002. Effect of freeze temperature on ice formation and long- term 1197 survival of the wooly bear caterpillar (Pyrrharctia isabella). J Insect Physiol 48, 113311981137.

1199Layne, J.R., Lee, R.E., Huang, J.L., 1990. Inoculation triggers freezing at high subzero 1200 temperatures in a freeze-tolerant frog (Rana sylvatica) and insect (Eurosta solidaginis). 1201 Can J Zool 68, 506-510.

1202Leather, S.R., Walters, K.F.A., Bale, J.S., 1993. The Ecology of Insect Overwintering. 1203 Cambridge University Press, Cambridge.

1204Lee, R.E., 1991. Principles of insect low temperature tolerance, in: Lee, R.E., Denlinger, D.L. 1205 (Eds.), Insects at Low Temperature. Chapman and Hall, New York, pp. 17-46. 
1206Lee, R.E., 2010. A primer on insect cold tolerance, in: Denlinger, D.L., Lee, R.E. (Eds.), Low

1207 Temperature Biology of Insects. Cambridge University Press, Cambridge, pp. 3-34.

1208Lee, R.E., Chen, C.-P., Denlinger, D.L., 1987. A rapid cold-hardening process in insects. Science 1209 238, 1415-1417.

1210Lee, R.E., Costanzo, J.P., Kaufman, P.E., Lee, M.R., Wyman, J.A., 1994. Ice-nucleating active

1211 bacteria reduce the cold-hardiness of the freeze-intolerant Colorado Potato Beetle

1212 (Coleoptera: Chrysomelidae). J Econ Entomol 87, 377-381.

1213Lee, R.E., Denlinger, D.L., 2010. Rapid cold-hardening: Ecological significance and

1214 underpinning mechanisms, in: Denlinger, D.L., Lee, R.E. (Eds.), Low temperature biology

1215 of insects. Cambridge University Press, Cambridge, pp. 35-58.

1216Lee, R.E., Hankison, S.J., 2003. Acquisition of freezing tolerance in early autumn and seasonal

1217 changes in gall water content influence inoculative freezing of gall fly larvae, Eurosta

1218 solidaginis (Diptera, Tephritidae). J Insect Physiol 49, 385-393.

1219Lee, R.E., Lewis, E.A., 1985. Effect of temperature and duration of exposure on tissue ice

1220 formation in the gall fly, Eurosta solidaginis (Diptera, Tephritidae). Cryo-Lett 7, 25-34.

1221Li, N., Zachariassen, K., 2007. Cold hardiness of insects distributed in the area of Siberian Cold

1222 Pole. Comp Biochem Physiol A 146, S156-S157.

1223Lighton, J.R.B., Turner, R.J., 2004. Thermolimit respirometry: an objective assessment of critical

1224 thermal maxima in two sympatric desert harvester ants, Pogonomyrmex rugosus and $P$.

1225 californicus. J Exp Biol 207, 1903-1913.

1226Macdonald, S.S., Rako, L., Batterham, P., Hoffmann, A.A., 2004. Dissecting chill coma recovery

1227 as a measure of cold resistance: evidence for a biphasic response in Drosophila

1228 melanogaster. J Insect Physiol 50, 695-700.

1229MacMillan, H.A., Sinclair, B.J., 2011a. Mechanisms underlying insect chill-coma. J Insect

1230 Physiol 57, 12-20.

1231MacMillan, H.A., Sinclair, B.J., 2011b. The role of the gut in insect chilling injury: cold-induced

1232 disruption of osmoregulation in the fall field cricket, Gryllus pennsylvanicus. J Exp Biol

$1233214,726-734$.

1234MacMillan, H.A., Walsh, J.P., Sinclair, B.J., 2009. The effects of selection for cold tolerance on

1235 cross-tolerance to other environmental stressors in Drosophila melanogaster. Insect Sci

$123616,263-276$. 
1237MacMillan, H.A., Williams, C.M., Staples, J.F., Sinclair, B.J., 2012a. Metabolism and energy 1238 supply below the critical thermal minimum of a chill-susceptible insect. J Exp Biol 215, $1239 \quad 1366-1372$.

1240Macmillan, H.A., Williams, C.M., Staples, J.F., Sinclair, B.J., 2012b. Reestablishment of ion 1241 homeostasis during chill-coma recovery in the cricket Gryllus pennsylvanicus. Proc Natl 1242 Acad Sci USA 109, 20750-20755.

1243Magiafoglou, A., Hoffmann, A.A., 2003. Cross-generation effects due to cold exposure in 1244 Drosophila serrata. Funct Ecol 17, 664-672.

1245Marshall, K.E., Sinclair, B.J., 2011. The sub-lethal effects of repeated freezing in the woolly bear 1246 caterpillar Pyrrharctia isabella. J Exp Biol 214, 1205-1212.

1247McArdle, B.H., 1996. Levels of evidence in studies of competition, predation, and disease. N Z J 1248 Ecol 20, 7-15.

1249Miller, L.K., 1969. Freezing tolerance in an adult insect. Science 166, 105-106.

1250Miller, L.K., 1978. Freezing tolerance in relation to cooling rate in an adult insect. Cryobiol 15, 1251 345-349.

1252Mori, N., Kimura, M.T., 2008. Selection for rapid and slow recovery from chill- and heat-coma 1253 in Drosophila melanogaster. Biol J Linnean Soc 95, 72-80.

1254Morrissey, R., Baust, J.G., 1976. The ontogeny of cold tolerance in the gall fly, Eurosta 1255 solidaginis. J Insect Physiol 22, 431-438.

1256Nedvěd, O., 1998. Modelling the relationship between cold injury and accumulated degree days 1257 in terrestrial arthropods. Cryo-Lett 19, 267-274.

1258Nedvěd, O., Hodková, M., Brunnhofer, V., Hodek, I., 1995. Simultaneous measurement of low

1259 temperature survival and supercooling in a sample of insects. Cryo-Lett 16, 108-113.

1260Nedvěd, O., Lavy, D., Verhoef, H.A., 1998. Modelling the time-temperature relationship in cold

1261 injury and effect of high-temperature interruptions on survival in a chill-sensitive 1262 collembolan. Funct Ecol 12, 816-824.

1263Nguyen, C., Bahar, M.H., Baker, G., Andrew, N.R., 2014. Thermal Tolerance Limits of 1264 Diamondback Moth in Ramping and Plunging Assays. PLoS One 9, e87535.

1265Nilson, T.N., Sinclair, B.J., Roberts, S.P., 2006. The effects of carbon dioxide anesthesia and 1266 anoxia on rapid cold-hardening and chill coma recovery in Drosophila melanogaster. J

1267 Insect Physiol 52, 1027-1033. 
1268Nunamaker, R.A., 1993. Rapid Cold-Hardening in Culicoides variipennis sonorensis (Diptera, 1269 Ceratopogonidae). J Med Entomol 30, 913-917.

1270Nyamukondiwa, C., Terblanche, J.S., Marshall, K.E., Sinclair, B.J., 2011. Basal cold but not heat 1271 tolerance constrains plasticity among Drosophila species (Diptera: Drosophilidae). J Evol 1272 Biol 24, 1927-1938.

1273Nylin, S., Gotthard, K., 1998. Plasticity in life-history traits. Annu Rev Entomol 43, 63-83. 1274Overgaard, J., Sørensen, J.G., 2008. Rapid thermal adaptation during field temperature variations 1275 in Drosophila melanogaster. Cryobiology 56, 159-162.

1276Palmer, C.M., Siebke, K., Yeates, D.K., 2004. Infrared video thermography: a technique for 1277 assessing cold adaptation in insects. Biotechniques 37, 212-217.

1278Pauli, J.N., Zuckerberg, B., Whiteman, J.P., Porter, W., 2013. The subnivium: a deteriorating 1279 seasonal refugium. Frontiers Ecol Env 11, 260-267.

1280Powell, S.J., Bale, J.S., 2004. Cold shock injury and ecological costs of rapid cold hardening in 1281 the grain aphid Sitobion avenae (Hemiptera : Aphididae). J Insect Physiol 50, 277-284. 1282Powell, S.J., Bale, J.S., 2005. Low temperature acclimated populations of the grain aphid 1283 Sitobion avenae retain ability to rapidly cold harden with enhanced fitness. J Exp Biol $1284 \quad 208,2615-2620$.

1285Powell, S.J., Bale, J.S., 2008. Intergenerational acclimation in aphid overwintering. Ecol 1286 Entomol 33, 95-100.

1287Rajamohan, A., Sinclair, B.J., 2008. Short-term hardening effects on survival of acute and 1288 chronic cold exposure by Drosophila melanogaster larvae. J Insect Physiol 54, 708-718. 1289Rajamohan, A., Sinclair, B.J., 2009. Hardening trumps acclimation in improving cold tolerance 1290 of Drosophila melanogaster larvae. Physiol Entomol 34, 217-223.

1291Ramløv, H., Westh, P., 1993. Ice formation in the freeze-tolerant alpine weta Hemideina maori 1292 Hutton (Orthoptera: Stenopelmatidae). Cryo-Lett 14, 169-176.

1293Ransberry, V.E., Macmillan, H.A., Sinclair, B.J., 2011. The relationship between chill-coma 1294 onset and recovery at the extremes of the thermal window of Drosophila melanogaster. 1295 Physiol Biochem Zool 84, 553-559.

1296Reis, M., Vieira, C.P., Morales-Hojas, R., Aguiar, B., Rocha, H., Schlotterer, C., Vieira, J., 2011. 1297 A Comparative Study of the Short Term Cold Resistance Response in Distantly Related 1298 Drosophila Species: The Role of regucalcin and Frost. PLoS One 6. 
1299Renault, D., Lalouette, L., 2012. Critical thermal minima of three sub-Antarctic insects from the 1300 French southern Indian Ocean islands. Antarct Sci 24, 43-44.

1301Renault, D., Salin, C., Vannier, G., Vernon, P., 1999. Survival and chill-coma in the adult lesser 1302 mealworm, Alphitobius diaperinus (Coleoptera: Tenebrionidae), exposed to low 1303 temperatures. J Therm Biol 24, 229-236.

1304Renault, D., Salin, C., Vannier, G., Vernon, P., 2002. Survival at low temperatures in insects: 1305 What is the ecological significance of the supercooling point? Cryolett 23, 217-228. 1306Renault, D., Vernon, P., Vannier, G., 2004. Comparing the freezing susceptibility of third-instar 1307 larvae of Gnorimus variabilis (Cetoniidae : Trichiinae) from three distant geographical 1308 regions. Can J Zool 82, 873-879.

1309Rezende, E.L., Tejedo, M., Santos, M., 2011. Estimating the adaptive potential of critical thermal 1310 limits: methodological problems and evolutionary implications. Funct Ecol 25, 111-121. 1311Ring, R.A., Riegert, P.W., 1991. A tribute to R. W. Salt, in: Lee, R.E., Denlinger, D.L. (Eds.), 1312 Insects at Low Temperature. Chapman and Hall, New York, pp. 3-16.

1313Salin, C., Renault, D., Vannier, G., Vernon, P., 2000. A sexually dimorphic response in 1314 supercooling temperature, enhanced by starvation, in the lesser mealworm Alphitobius 1315 diaperinus (Coleoptera : Tenebrionidae). J Therm Biol 25, 411-418.

1316Salt, R.W., 1961. Principles of insect cold hardiness. Ann Rev Entomol 6, 55-74. 1317Salt, R.W., 1966. Effect of cooling rate on the freezing temperatures of supercooled insects. Can 1318 J Zool 44, 655-659.

1319Santos, M., Castaneda, L.E., Rezende, E.L., 2011. Making sense of heat tolerance estimates in 1320 ectotherms: lessons from Drosophila. Funct Ecol 25, 1169-1180.

1321Scharf, I., Galkin, N., Halle, S., 2015. Disentangling the Consequences of Growth Temperature 1322 and Adult Acclimation Temperature on Starvation and Thermal Tolerance in the Red Flour 1323 Beetle. Evol Biol 42, 54-62.

1324Sformo, T., Kohl, F., McIntyre, J., Kerr, P., Duman, J.G., Barnes, B.M., 2009. Simultaneous 1325 freeze tolerance and avoidance in individual fungus gnats, Exechia nugatoria. J Comp 1326 Physiol B 179, 897-902.

1327Sformo, T., McIntyre, J., Walters, K.R., Barnes, B.M., Duman, J., 2011. Probability of freezing in 1328 the freeze-avoiding beetle larvae Cucujus clavipes puniceus (Coleoptera: Cucujidae) from 1329 interior Alaska. J Insect Physiol 57, 1170-1177. 
1330Shimada, K., Riihimaa, A., 1988. Cold acclimation, inoculative freezing and slow cooling:

1331 essential factors contributing to the freezing-tolerance in diapausing larvae of Chymomyza 1332 costata (Diptera: Drosophilidae). Cryo-Lett 9, 5-10.

1333Shuman, D., Coffelt, J.A., Weaver, D.K., 1996. A computer-based electronic fall-through probe

1334 insect counter for monitoring infestation in stored products. Trans ASAE 39, 1773-1780.

1335Sinclair, B., Klok, C., Scott, M., Terblanche, J., Chown, S., 2003a. Diurnal variation in

1336 supercooling points of three species of Collembola from Cape Hallett, Antarctica. J Insect

1337 Physiol 49, 1049-1061.

1338Sinclair, B., Vernon, P., Klok, C., Chown, S., 2003b. Insects at low temperatures: an ecological 1339 perspective. Trends Ecol Evol 18, 257-262.

1340Sinclair, B.J., 1997. Seasonal variation in freezing tolerance of the New Zealand alpine

1341 cockroach Celatoblatta quinquemaculata. Ecol Entomol 22, 462-467.

1342Sinclair, B.J., 1999. Insect cold tolerance: How many kinds of frozen? Eur J Entomol 96, 1571343164.

1344Sinclair, B.J., 2001. Field ecology of freeze tolerance: interannual variation in cooling rates,

1345 freeze-thaw and thermal stress in the microhabitat of the alpine cockroach Celatoblatta 1346 quinquemaculata. Oikos 93, 286-293.

1347Sinclair, B.J., Chown, S.L., 2003. Rapid responses to high temperature and desiccation but not to 1348 low temperature in the freeze tolerant sub-Antarctic caterpillar Pringleophaga marioni 1349 (Lepidoptera, Tineidae). J Insect Physiol 49, 45-52.

1350Sinclair, B.J., Chown, S.L., 2005. Climatic variability and hemispheric differences in insect cold 1351 tolerance: support from southern Africa. Funct Ecol 19, 214-221.

1352Sinclair, B.J., Chown, S.L., 2006. Rapid cold-hardening in a Karoo beetle, Afrinus sp. Physiol

1353 Entomol 31, 98-101.

1354Sinclair, B.J., Gibbs, A.G., Lee, W.K., Rajamohan, A., Roberts, S.P., Socha, J.J., 2009.

1355 Synchrotron X-Ray Visualisation of Ice Formation in Insects during Lethal and Non-

1356 Lethal Freezing. Plos One 4, E8259.

1357Sinclair, B.J., Klok, C.J., Chown, S.L., 2004. Metabolism of the sub-Antarctic caterpillar 1358 Pringleophaga marioni during cooling, freezing and thawing. J Exp Biol 207, 1287-1294. 
1359Sinclair, B.J., Klok, C.J., Scott, M.B., Terblanche, J.S., Chown, S.L., 2003c. Diurnal variation in 1360 supercooling points of three species of Collembola from Cape Hallett, Antarctica. J Insect 1361 Physiol 49, 1049-1061.

1362Sinclair, B.J., Roberts, S.P., 2005. Acclimation, shock and hardening in the cold. J Therm Biol $1363 \quad 30,557-562$.

1364Sinclair, B.J., Sjursen, H., 2001. Cold tolerance of the Antarctic springtail Gomphiocephalus 1365 hodgsoni (Collembola, Hypogastruridae). Antarct Sci 13, 271-279.

1366Sinclair, B.J., Stinziano, J.R., Williams, C.M., MacMillan, H.A., Marshall, K.E., Storey, K.B., 1367 2013. Real-time measurement of metabolic rate during freezing and thawing of the wood 1368 frog, Rana sylvatica: implications for overwinter energy use. J Exp Biol 216, 292-302. 1369Sinclair, B.J., Terblanche, J.S., Scott, M.B., Blatch, G., Klok, C.J., Chown, S.L., 2006a.

1370 Environmental physiology of three species of Collembola at Cape Hallett, North Victoria 1371 Land, Antarctica. J Insect Physiol 52, 29-50.

1372Sinclair, B.J., Terblanche, J.S., Scott, M.B., Blatch, G.L., Klok, C.J., Chown, S.L., 2006b.

1373 Environmental physiology of three species of Collembola at Cape Hallett, North Victoria 1374 Land, Antarctica. J Insect Physiol 52, 29-50.

1375Sinclair, B.J., Williams, C.M., Terblanche, J.S., 2012. Variation in thermal performance among 1376 insect populations. Physiol Biochem Zool 85, 594-606.

1377Sinclair, B.J., Worland, M.R., Wharton, D.A., 1999. Ice nucleation and freezing tolerance in New 1378 Zealand alpine and lowland weta, Hemideina spp. (Orthoptera; Stenopelmatidae). Physiol 1379 Entomol 24, 56-63.

1380Sisodia, S., Singh, B.N., 2010. Influence of developmental temperature on cold shock and chill 1381 coma recovery in Drosophila ananassae: Acclimation and latitudinal variations among 1382 Indian populations. J Therm Biol 35, 117-124.

1383Sisodia, S., Singh, B.N., 2012. Experimental evidence for nutrition regulated stress resistance in 1384 Drosophila ananassae. PloS one 7, e46131.

1385Slabber, S., Chown, S.L., 2004. Thermal tolerance and cold hardiness strategy of the sub1386 Antarctic psocid Antarctopsocus jeanneli Badonnel. Polar Biol 28, 56-61. 1387Slabber, S., Chown, S.L., 2005. Differential responses of thermal tolerance to acclimation in the 1388 sub-Antarctic rove beetle Halmaeusa atriceps. Physiol Entomol 30, 195-204. 
1389Sobek-Swant, S., Crosthwaite, J.C., Lyons, D.B., Sinclair, B.J., 2012. Could phenotypic 1390 plasticity limit an invasive species? Incomplete reversibility of mid-winter deacclimation 1391 in emerald ash borer. Biol Invasions 14, 115-125.

1392Sømme, L., 1982. Supercooling and winter survival in terrestrial arthropods. Comp Biochem 1393 Physiol 73A, 519-543.

1394Sømme, L., 1995. Invertebrates in Hot and Cold Arid Environments. Springer-Verlag, Berlin. 1395Sømme, L., 1996. The effect of prolonged exposures at low temperatures in insects. Cryo-Lett $1396 \quad 17,341-346$.

1397Sømme, L., 2000. The history of cold hardiness research in terrestrial arthropods. CryoLett 21, $1398 \quad 289-296$.

1399Sømme, L., Davidson, R.L., Onore, G., 1996. Adaptations of insects at high altitudes of 1400 Chimborazo, Ecuador. Eur J Entomol 93, 313-318.

1401Sømme, L., Strømme, A., Zachariassen, K.E., 1993. Notes on the ecology and physiology of the 1402 Antarctic oribatid mite Maudheimia wilsoni. Polar Res 12, 21-25.

1403Sømme, L., Zachariassen, K.E., 1981. Adaptations to low temperature in high altitude insects 1404 from Mount Kenya. Ecol Entomol 6, 199-204.

1405Sørensen, J.G., Holmstrup, M., 2011. Cryoprotective dehydration is widespread in Arctic 1406 springtails. J Insect Physiol 57, 1147-1153.

1407Storey, K.B., Storey, J.M., 1991. Biochemistry of cryoprotectants, in: Lee, R.E., Denlinger, D.L. 1408 (Eds.). Chapman and Hall, New York, pp. 64-93.

1409Storey, K.B., Storey, J.M., 2013. Molecular Biology of Freezing Tolerance. Compr Physiol 3, $1410 \quad 1283-1308$.

1411Strachan, L.A., Tarnowski-Garner, H.E., Marshall, K.E., Sinclair, B.J., 2011. The Evolution of 1412 Cold Tolerance in Drosophila Larvae. Physiol Biochem Zool 84, 43-53.

1413Tattersall, G.J., Sinclair, B.J., Withers, P.C., Fields, P.A., Seebacher, F., Cooper, C.E., Maloney, 1414 S.K., 2012. Coping with Thermal Challenges: Physiological Adaptations to Environmental 1415 Temperatures. Compr Physiol 2, 2151-2202.

1416Teets, N.M., Denlinger, D.L., 2013. Physiological mechanisms of seasonal and rapid cold1417 hardening in insects. Physiol Entomol 38, 105-116. 
1418Telonis-Scott, M., Hallas, R., McKechnie, S.W., Wee, C.W., Hoffmann, A.A., 2009. Selection for 1419 cold resistance alters gene transcript levels in Drosophila melanogaster. J Insect Physiol $1420 \quad 55,549-555$.

1421Terblanche, J.S., Deere, J.A., Clusella-Trullas, S., Janion, C., Chown, S.L., 2007. Critical thermal 1422 limits depend on methodological context. Proc R Soc B 274, 2935-2942.

1423Terblanche, J.S., Hoffmann, A.A., Mitchell, K.A., Rako, L., le Roux, P.C., Chown, S.L., 2011.

1424 Ecologically relevant measures of tolerance to potentially lethal temperatures. J Exp Biol $1425214,3713-3725$.

1426Terblanche, J.S., Klok, C.J., Krafsur, E.S., Chown, S.L., 2006. Phenotypic plasticity and 1427 geographic variation in thermal tolerance and water loss of the tsetse Glossina pallidipes 1428 (Diptera : Glossinidae): Implications for distribution modelling. Amer J Trop Med Hyg 74, 1429 786-794.

1430Tomcala, A., Tollarova, M., Overgaard, J., Simek, P., Koštál, V., 2006. Seasonal acquisition of 1431 chill tolerance and restructuring of membrane glycerophospholipids in an overwintering 1432 insect: triggering by low temperature, desiccation and diapause progression. J Exp Biol 1433 209, 4102-4114.

1434Udaka, H., Sinclair, B.J., 2014. The overwintering biology of the acorn weevil, Curculio 1435 glandium in southwestern Ontario. J Therm Biol 44, 103-109.

1436van der Merwe, M., Chown, S.L., Smith, V.R., 1997. Thermal tolerance limits in six weevil 1437 species (Coleoptera, Curculionidae) from sub-Antarctic Marion Island. Polar Biol 18, 3311438336.

1439van Dooremalen, C., Berg, M.P., Ellers, J., 2013. Acclimation responses to temperature vary with 1440 vertical stratification: implications for vulnerability of soil-dwelling species to extreme 1441 temperature events. Global Change Biol 19, 975-984.

1442Voituron, Y., Mouquet, N., de Mazancourt, C., Clobert, J., 2002. To freeze or not to freeze? An 1443 evolutionary perspective on the cold-hardiness strategies of overwintering ectotherms. 1444 Amer Nat 160, 255-270.

1445Watanabe, M., Tanaka, K., 1998. Adult diapause and cold hardiness in Aulacophora nigripennis 1446 (Coleoptera : Chrysomelidae). J Insect Physiol 44, 1103-1110.

1447Wharton, D.A., Rowland, J.J., 1984. A thermoelectric cooling stage for the measurement of the 1448 supercooling points of microscopic organisms. J Microsc 134, 299-305. 
1449Williams, C.M., Hellmann, J.J., Sinclair, B.J., 2012. Lepidopteran species differ in susceptibility 1450 to winter warming. Clim Res 53, 119-130.

1451Williams, C.M., Henry, H.A.L., Sinclair, B.J., 2015. Cold truths: how winter drives responses of 1452 terrestrial organisms to climate change. Biol Rev 90, 214-235.

1453Williams, C.M., Nicolai, A., Ferguson, L.V., Bernards, M.A., Hellmann, J.J., Sinclair, B.J., 2014. 1454 Cold hardiness and deacclimation of overwintering Papilio zelicaon pupae. Comp 1455 Biochem Physiol A 178, 51-58.

1456Worland, M.R., Convey, P., 2001. Rapid cold hardening in Antarctic microarthropods. Funct Ecol $1457 \quad 15,515-524$.

1458Worland, M.R., Leinaas, H.P., Chown, S.L., 2006. Supercooling point frequency distributions in 1459 Collembola are affected by moulting. Funct Ecol 20, 323-329.

1460Zachariassen, K.E., 1985. Physiology of cold tolerance in insects. Physiol Rev 65, 799-832. 1461Zachariassen, K.E., 1991. The water relations of overwintering insects, in: Lee, R.E., Denlinger, 1462 D.L. (Eds.). Chapman and Hall, New York, pp. 47-63.

1463Zachariassen, K.E., Kristiansen, E., 2000. Ice nucleation and antinucleation in nature. Cryobiol $1464 \quad 41,257-279$.

1465Zachariassen, K.E., Kristiansen, E., Pedersen, S.A., 2004. Inorganic ions in cold-hardiness. 1466 Cryobiol 48, 126-133.

1467 


\section{Figure Captions}

1469 Figure 1. Classifications of insect cold tolerance. Insect cold tolerance is divided into

1470 three main classifications, based on survival of low temperatures and extracellular ice

1471 formation. Chill-susceptible insects die of cold exposure unrelated to freezing, whereas

1472 freeze-avoiding insects maintain their body fluids in a supercooled state and die when ice

1473 formation occurs (i.e. at the supercooling point). Freeze-tolerant insect are able to withstand

1474 extracellular ice formation. Adapted from Lee (2010).

1476 Figure 2. Attaching thermocouples to insects. A. For small insects, thermocouples are

1477 secured to the insect using a thin layer of vacuum grease. B. For medium-sized and/or active

1478 insects, a piece of cotton can be inserted into the vessel (pipette tip, microtube) to

1479 immobilize the insect near the tip and secure the thermocouple in contact with the insect. C.

1480 For large and highly active insects, the thermocouple can be secured in contact with the

1481 insect using adhesive, such as adhesive putty.

1483 Figure 3. Exotherms from insects of different sizes. A: an exotherm from a small insect, a

1484 c. $1.6 \mathrm{mg}$ fruit fly (Drosophila suzukii). B: an exotherm from a larger insect, a c. $153 \mathrm{mg}$

1485 cricket (Gryllus veletis). C: an exotherm from Gryllus veletis (135 mg) preceded by an

1486 artifact caused by condensation in or on the chamber. All measurements were made using

148736 AWG type-T thermocouples. 
1489 Figure 4. Supercooling point distributions from two different species of Antarctic

1490 Collembola. A. A bimodal distribution of supercooling points of Cryptopygus

1491 cisantarcticus (Isotomidae). B. A unimodal distribution of supercooling points of Friesea

1492 grisea (Neanuridae). Redrawn from Sinclair et al., (2006a).

1493

1494 Figure 5. Time course of ice accumulation in adult Hemideina maori held at $-5^{\circ} \mathrm{C}$.

1495 Redrawn after Ramløv and Westh (1993).

1497 Figure 6. Hallmarks of cryoprotective dehydration in insects. A. Characteristically,

1498 insect body water content and the melting point of the insect will decrease as the ambient

1499 temperature decreases. Redrawn from Sinclair et al. (2003b). B. In the collembolan

1500 Onychiurus arcticus, the melting point of the body fluids does not change over time if the

1501 insect is held over water, whereas the melting point decreases over time and mimics that of

1502 the ambient temperature when the insect is held over ice. The dotted line represents the

1503 average melting point over ice and the solid line represents the ambient temperature.

1504 Redrawn from Holmstrup et al. (2002).

1506 Figure 7. Determining lethal temperatures. A. Estimate of the lower lethal temperature of

1507 Diamesa mendotae based on survival of subzero temperatures. Flies were cooled to subzero

1508 temperatures and immediately re-warmed upon reaching the designated temperature.

1509 Survival was recorded 24h following exposure. The lower lethal temperature was estimated

1510 to be $-21.5^{\circ} \mathrm{C}$. Redrawn from Bouchard et al. (2006). B. Survival of Eldana saccharina 
1511 larvae exposed to low temperatures. Larvae were directly exposed to temperatures ranging 1512 from $10^{\circ} \mathrm{C}$ to $-10{ }^{\circ} \mathrm{C}$ for $2 \mathrm{~h}$. Solid line represents the fitted logistic regression model. Re1513 drawn from Kleynhans et al. (2014).

1515 Figure 8. Variation in chill-coma recovery time depending on entry (A, B) and exit (C,

1516 D) from chill coma. A. CCR of insects $x, y$, and $\mathrm{z}$ are equal, because equilibrium is reached

1517 during time spent in chill coma. B. CCR of $x$ is less than $y($ and $z$ ), because the rate of entry

1518 into chill coma did not allow for equilibrium to be reached before recovery began. C. CCR

1519 of $y$ is greater than $\mathrm{x}$, despite the same time spent in chill coma, because the equilibrium of $\mathrm{y}$

1520 is lower than that of $\mathrm{x}$. D. The CCR of $\mathrm{y}$ is greater than $\mathrm{x}$ because recovery occurs at a

1521 slower rate. $\mathrm{E}_{\mathrm{k}}=$ membrane equilibrium potential of $\mathrm{K}^{+}$.

1523 Figure 9. Examples of temperature \& photoperiod manipulations used induce

1524 phenotypic plasticity in the laboratory. A. Acute exposure to low temperatures to induce a

1525 rapid cold-hardening response. B. A 5-10 day exposure to low temperatures and short day-

1526 length. C. Development under low temperatures and short day-length. D. Exposure to

1527 gradually declining temperature and photoperiod. $\mathbf{L}=$ light, $\mathbf{D}=$ dark.

1529 Figure 10. Recommended work flow to determine cold tolerance strategies and lower 1530 thermal limits for investigating overwintering potential and comparisons of cold tolerance 1531 among groups. A. Work flow for determining cold tolerance strategy and overwintering 1532 potential. B. Work flow for using lower limits as a physiological comparison among groups. 


\section{Tables}

1534 Table 1. Cold tolerance strategies of insects as determined by survival of internal ice

1535 formation. Chill-susceptible insects die of injuries unrelated to freezing, freeze-avoidant

1536 insects die upon internal ice formation, and freeze-tolerant species are able to survive

1537 internal ice formation.

1538

\begin{tabular}{|c|c|c|}
\hline 1539 & Internal ice formed & No internal ice formed \\
\hline 1540Alive & Freeze-tolerant & $\begin{array}{l}\text { Chill-susceptible or freeze- } \\
\text { avoidant }\end{array}$ \\
\hline 1542 Dead & $\begin{array}{l}\text { Chill-susceptible or freeze- } \\
\text { avoidant }\end{array}$ & Chill-susceptible \\
\hline
\end{tabular}

$154 \overline{3}$

1544 
1545 Table 2. Cold tolerance strategies of insects as determined by survival following

1546 exposure to the supercooling point. When half of the insects have reached their

1547 supercooling point during cooling, all insects are returned to warm conditions to monitor

1548 survival. This provides a group of insects that experienced freezing, and a group that did

1549 not, while maintaining exposure to approximately the same low temperature. Survival

1550 following freezing indicates freeze-tolerance, whereas survival until freezing is reached

1551 indicates freeze-avoidance. Mortality before the supercooling point is reached indicates

1552 chill-susceptibility. Drosophila suzukii: Jakobs et al. (2015); Reticulitermes flavipes: Clarke

1553 et al. (2013); Perisphaeria sp.: Sinclair and Chown (2005).

1554

\begin{tabular}{llcllll}
\hline Species & \multicolumn{2}{l}{ Drosophila suzukii } & \multicolumn{2}{l}{ Reticulitermes flavipes } & \multicolumn{2}{l}{ Perisphaeria sp. } \\
\hline $\begin{array}{l}\text { Physiological } \\
\text { state }\end{array}$ & Unfroze & Frozen & Unfroze & Frozen & Unfroze & Frozen \\
Survival & $0 \%$ & $0 \%$ & $\mathrm{n}$ & & $\mathrm{n}$ & \\
Strategy & \multicolumn{2}{l}{ Chill-susceptible } & \multicolumn{2}{l}{ Freeze-avoidant } & \multicolumn{2}{l}{ Freeze-tolerant } \\
\hline
\end{tabular}

$155 \overline{5}$ 
1556 Table 3. Examples of time and temperature treatments for measuring chill coma

1557 recovery. Time and temperature exposures often vary widely between studies and labs, and

1558 will depend on the low-temperature tolerance of the species of interest.

1559

\begin{tabular}{llll}
\hline Study & Study species & Time (h) & Temperature \\
\hline Andersen et al. (2015b) & Drosophila spp. & 2 & $-2{ }^{\circ} \mathrm{C}$ \\
Findsen et al. (2013) & Locusta migratoria & 2 & $-4{ }^{\circ} \mathrm{C}$ \\
(Ransberry et al., 2011) & Drosophila melanogaster & 6 & $0{ }^{\circ} \mathrm{C}$ \\
$\begin{array}{l}\text { Coello Alvarado et al. } \\
\text { (2015) }\end{array}$ & $\begin{array}{l}\text { Gryllus veletis and Gryllus } \\
\text { pennsylvanicus }\end{array}$ & 12 & $0{ }^{\circ} \mathrm{C}$ \\
David et al. (2003) & $\begin{array}{l}\text { Drosophila subobscura (four } \\
\text { populations) }\end{array}$ & 16 & $-7{ }^{\circ} \mathrm{C}$ to $+2{ }^{\circ} \mathrm{C}$ \\
& & &
\end{tabular}


1562 Table 4. Cues and conditions for inducing phenotypic plasticity in insect cold tolerance.

Cue/Condition

Shortened

Photoperiod

\section{Changes in diet}

\section{Maternal Effects}

Acute decrease

in temperature

Rapid cold

hardening

Slow cooling

Heat shock

\section{Acclimation}

\section{Short (5-10 d) Halmaeusa atriceps were held at $5{ }^{\circ} \mathrm{C}$ for $7 \mathrm{~d}$}

Slow decrease

Acclimation to $15^{\circ} \mathrm{C}$ for $1 \mathrm{wk}$, followed by $6^{\circ} \mathrm{C}$ for $1 \mathrm{wk}$, extends survival of Drosophila melanogaster at $0^{\circ} \mathrm{C}$

Ecologically relevant

Example

Shortened photoperiod (12 hours of light, 12 hours of dark) triggers increased cold-hardiness in

Aulacophoro nigripennis (Coleoptera: Chrysomelidae)

Drosophila ananassae larvae reared on carbohydrate-rich food are more cold- tolerant.

Chymomyza costata larvae reared on a proline-rich diet are more freeze-tolerant

The $\mathrm{LT}_{50}$ of Sitobion avenae offspring is lower if adults are reared at $10^{\circ} \mathrm{C}$, compared to $20^{\circ} \mathrm{C}$.

Exposure to $5^{\circ} \mathrm{C}$ for 30 min increases survival of Drosophila melanogaster at $-5^{\circ} \mathrm{C}$

$10 \mathrm{~min}$ to $1 \mathrm{hr}$ exposure to $0^{\circ} \mathrm{C}$ increases the freezing tolerance of Sarcophaga crassipalpis

Cooling at $0.05^{\circ} \mathrm{C} / \mathrm{min}$ or $0.1^{\circ} \mathrm{C} / \mathrm{min}$ increased survival of Drosophila melanogaster held at $-7^{\circ} \mathrm{C}$ for one hour

Exposure to $34{ }^{\circ} \mathrm{C}$ for $40-80$ min increases survival of Drosophila melanogaster larvae at $0{ }^{\circ} \mathrm{C}$

Heat pre-treatment $\left(36.5^{\circ} \mathrm{C}, 1 \mathrm{hr}\right)$ increased cold tolerance in larvae of Drosophila melanogaster

Lepidopteran pupae were exposed to fluctuating temperatures of $14: 10^{\circ} \mathrm{C}$ day: night for 2 wk, followed by $10: 6^{\circ} \mathrm{C}$ day: night for $2 \mathrm{wk}$, followed by a constant $6^{\circ} \mathrm{C}$

\section{Reference}

(Watanabe and Tanaka 1998)

(Sisodia and Singh, 2012)

(Koštál et al., 2011b)

(Powell and Bale, 2008)

(Czajka and Lee, 1990)

(Chen et al., 1987)

(Kelty and Lee, 1999)

(Burton et al., 1988)

(Rajamohan and Sinclair, 2008)

(Slabber and Chown,

(Koštál et al., 2011a)

(Williams et al., 2012) 


\begin{tabular}{|c|c|c|}
\hline $\begin{array}{l}\text { Rearing } \\
\text { temperature }\end{array}$ & Three generations of Sitobion avenae were reared at $10^{\circ} \mathrm{C}$ & (Powell and Bale, 2005) \\
\hline
\end{tabular}

\section{3}

Table 5. Requirements and considerations for measurements of cold tolerance in insects

1565

\begin{tabular}{|c|c|c|c|c|c|}
\hline Measurement & $\begin{array}{l}\text { Equipment } \\
\text { requirements }\end{array}$ & Ecological relevance & Constraints & Advantages & $\begin{array}{l}\text { Typical } \\
\text { sample size }\end{array}$ \\
\hline $\begin{array}{l}\text { Chill coma recovery } \\
\text { (CCR) } \\
\text { Section } 5.2\end{array}$ & $\begin{array}{l}\text { Simple cooling } \\
\text { Simple constant } \\
\text { temperature }\end{array}$ & Unclear & $\begin{array}{l}\text { Active/mobile } \\
\text { insects only } \\
\text { Cannot compare } \\
\text { among studies }\end{array}$ & $\begin{array}{l}\text { Easy to measure } \\
\text { Can be compared among treatment } \\
\text { groups }\end{array}$ & $10-20$ \\
\hline $\begin{array}{l}\text { Critical thermal } \\
\text { minimum }\left(\mathbf{C T}_{\min }\right) \\
\text { Section } 5.1\end{array}$ & $\begin{array}{l}\text { Controlled cooling } \\
\text { Temperature } \\
\text { measurement }\end{array}$ & $\begin{array}{l}\text { Moderate-high: sets the } \\
\text { lower limit for activity }\end{array}$ & $\begin{array}{l}\text { Continual } \\
\text { observation } \\
\text { Active/mobile } \\
\text { insects only }\end{array}$ & $\begin{array}{l}\text { Correlated to other measures of cold } \\
\text { tolerance (e.g. in Drosophila) } \\
\text { Can be compared among species and } \\
\text { treatments } \\
\text { Can be compared to ambient } \\
\text { temperatures }\end{array}$ & $10-20$ \\
\hline $\begin{array}{l}\text { Supercooling point } \\
\text { (SCP) } \\
\text { Section } 2.3\end{array}$ & $\begin{array}{l}\text { Controlled cooling } \\
\text { Temperature } \\
\text { measurement }\end{array}$ & $\begin{array}{l}\text { High if insect is freeze- } \\
\text { avoidant } \\
\text { Important threshold for } \\
\text { freeze-tolerant insects } \\
\text { No relevance if insect } \\
\text { is chill-susceptible }\end{array}$ & $\begin{array}{l}\text { Must consider } \\
\text { sources of ice } \\
\text { nucleation }\end{array}$ & $\begin{array}{l}\text { High throughput } \\
\text { Can be compared among species and } \\
\text { treatments } \\
\text { Can be compared to ambient } \\
\text { temperatures }\end{array}$ & $20-50$ \\
\hline $\begin{array}{l}\text { Cold tolerance } \\
\text { strategy } \\
\text { Section } 3.1\end{array}$ & $\begin{array}{l}\text { Controlled cooling } \\
\text { Temperature } \\
\text { measurement }\end{array}$ & None & $\begin{array}{l}\text { Does not predict } \\
\text { thermal limits }\end{array}$ & $\begin{array}{l}\text { Informs interpretation of other } \\
\text { measurements }\end{array}$ & $8-10$ \\
\hline $\begin{array}{l}\text { Lower lethal } \\
\text { temperature (LLT) } \\
\text { Section } 4.1\end{array}$ & $\begin{array}{l}\text { Controlled cooling } \\
\text { Temperature } \\
\text { measurement }\end{array}$ & High & Time-consuming & Directly relevant to the real world & $\begin{array}{l}5-10 \text { groups } \\
\text { of } 5-10\end{array}$ \\
\hline $\begin{array}{l}\text { Discriminating } \\
\text { temperature }\end{array}$ & $\begin{array}{l}\text { Simple, constant } \\
\text { temperature }\end{array}$ & $\begin{array}{l}\text { Moderate: predicts } \\
\text { survival at this }\end{array}$ & $\begin{array}{l}\text { Must determine } \\
\text { appropriate }\end{array}$ & $\begin{array}{l}\text { High throughput } \\
\text { With careful choice of temperature, can }\end{array}$ & $\begin{array}{l}\text { Groups of } 5- \\
10 \text { with or }\end{array}$ \\
\hline
\end{tabular}


temperature in

advance

be ecologically relevant.

groups/treatments

without

replicate

groups

$156 \overline{6}$ 


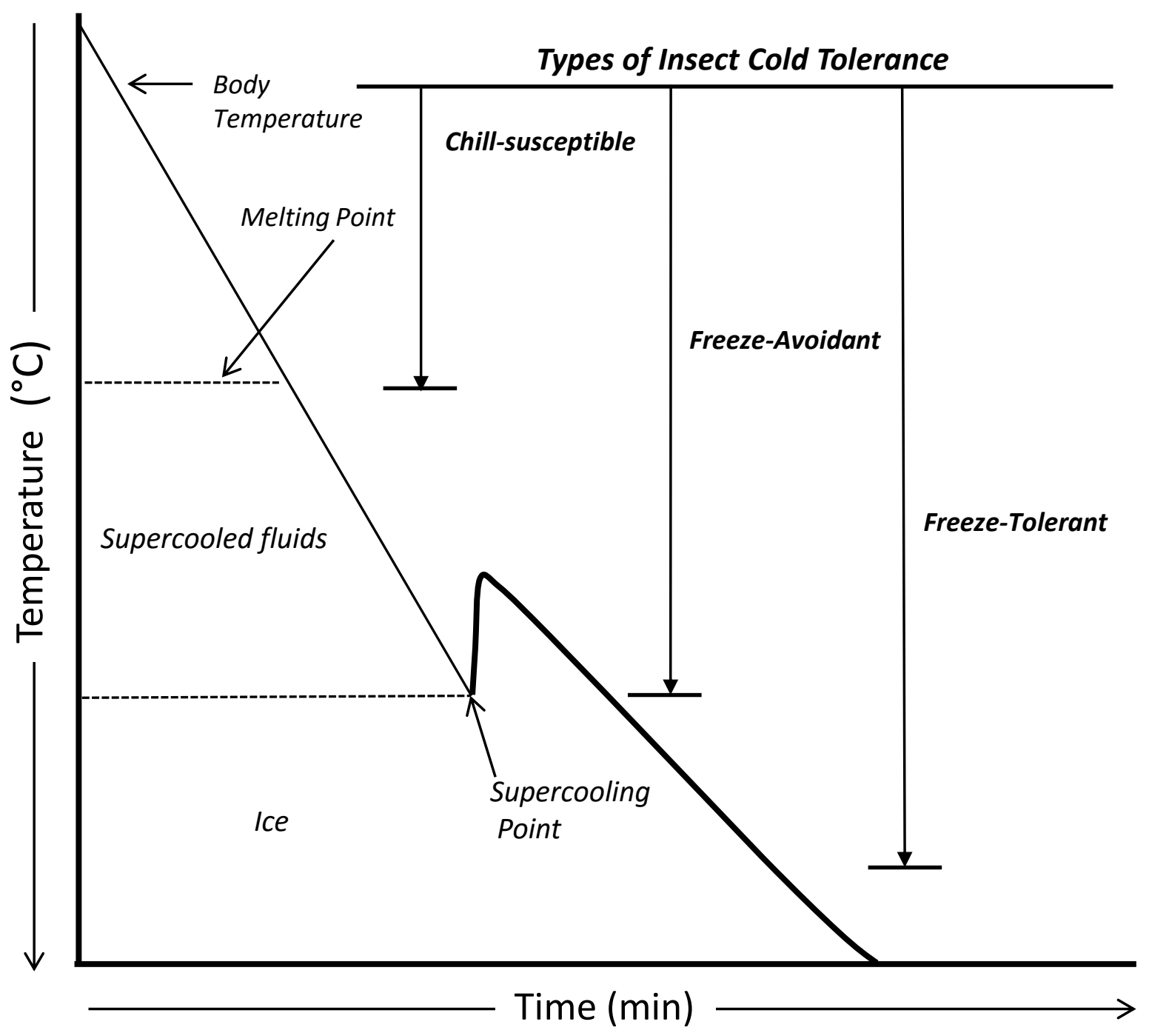




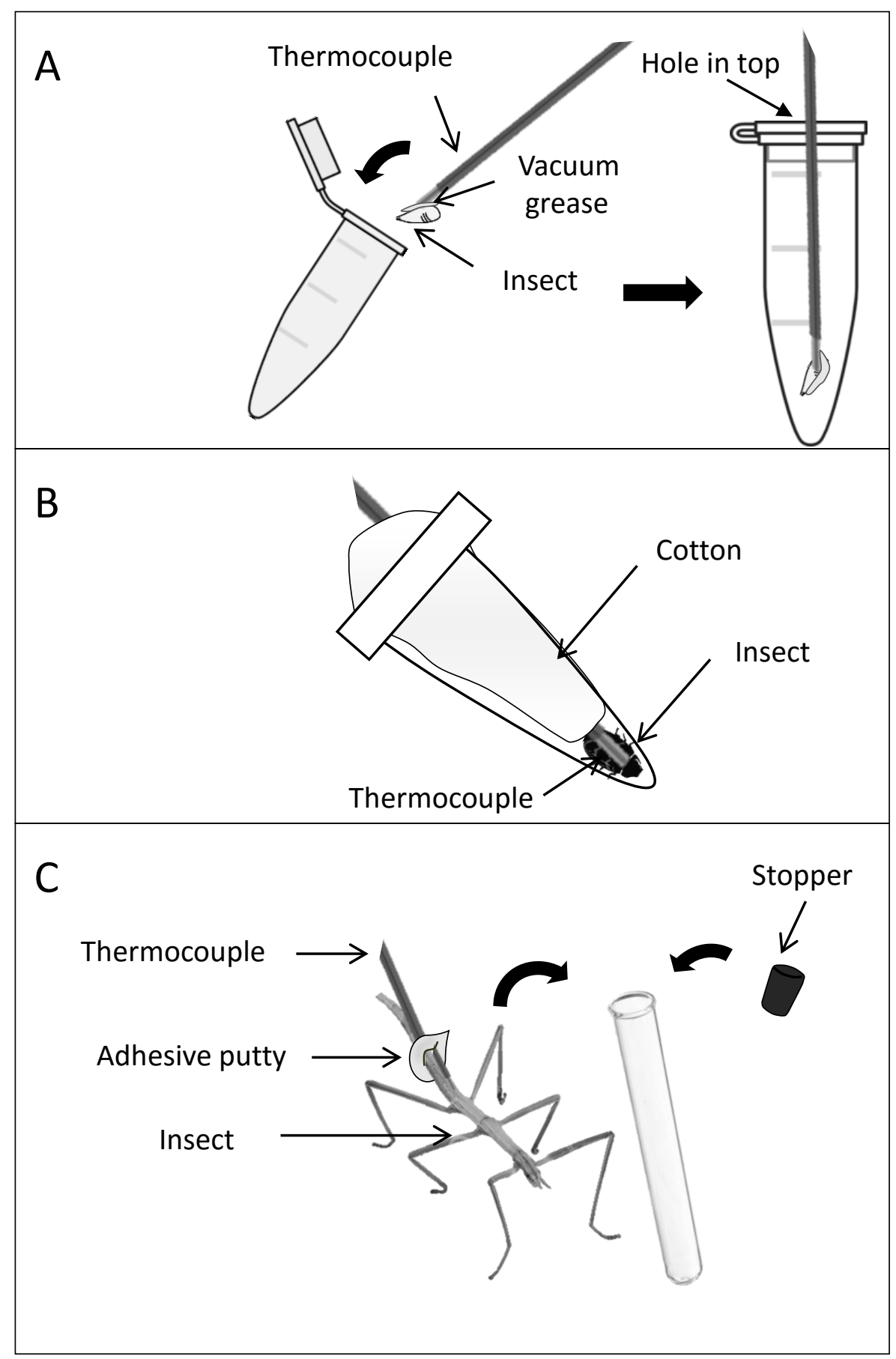



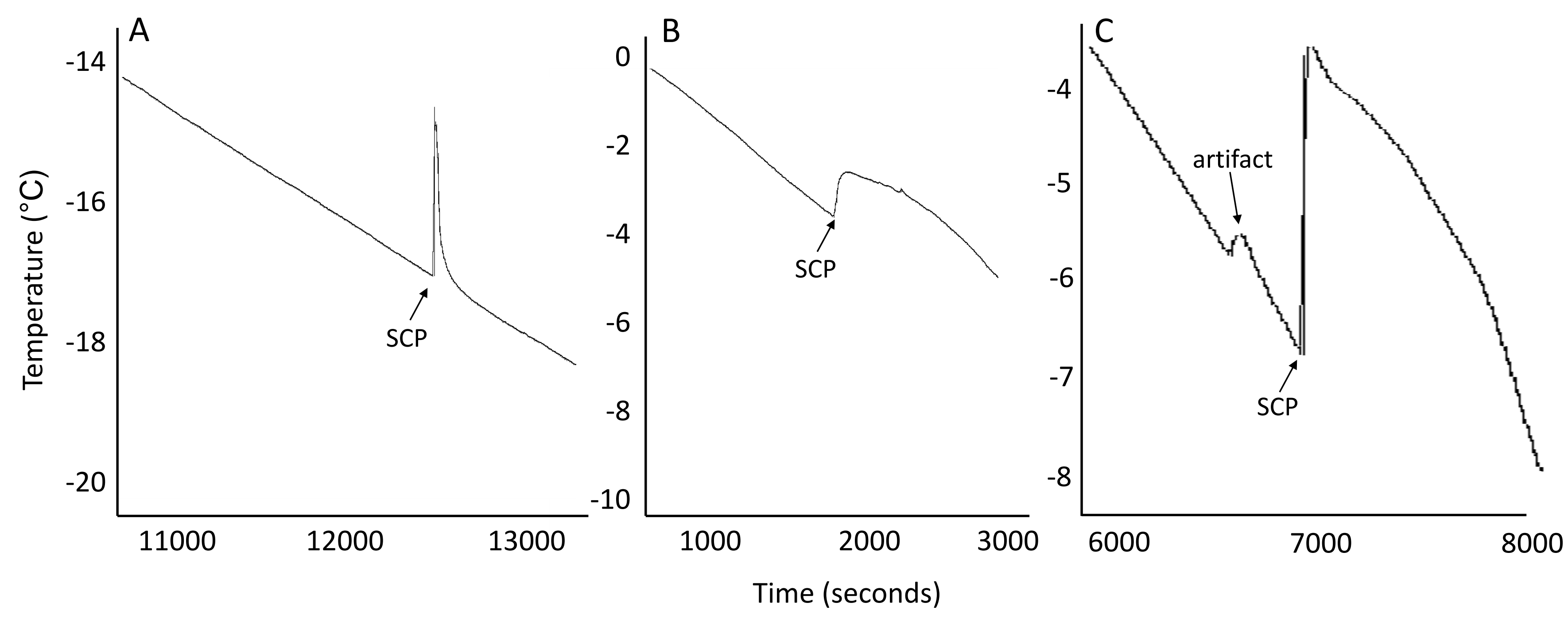

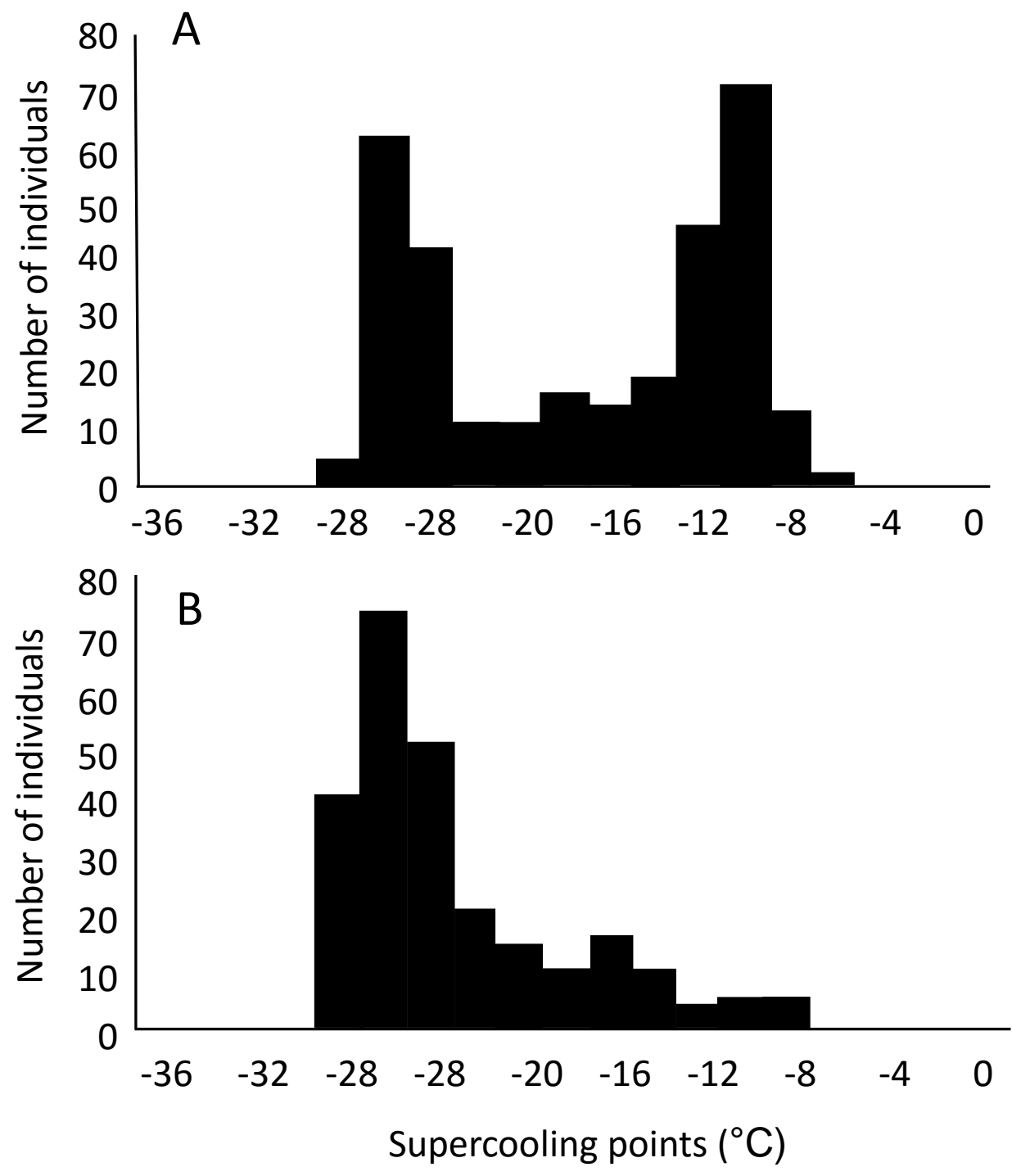


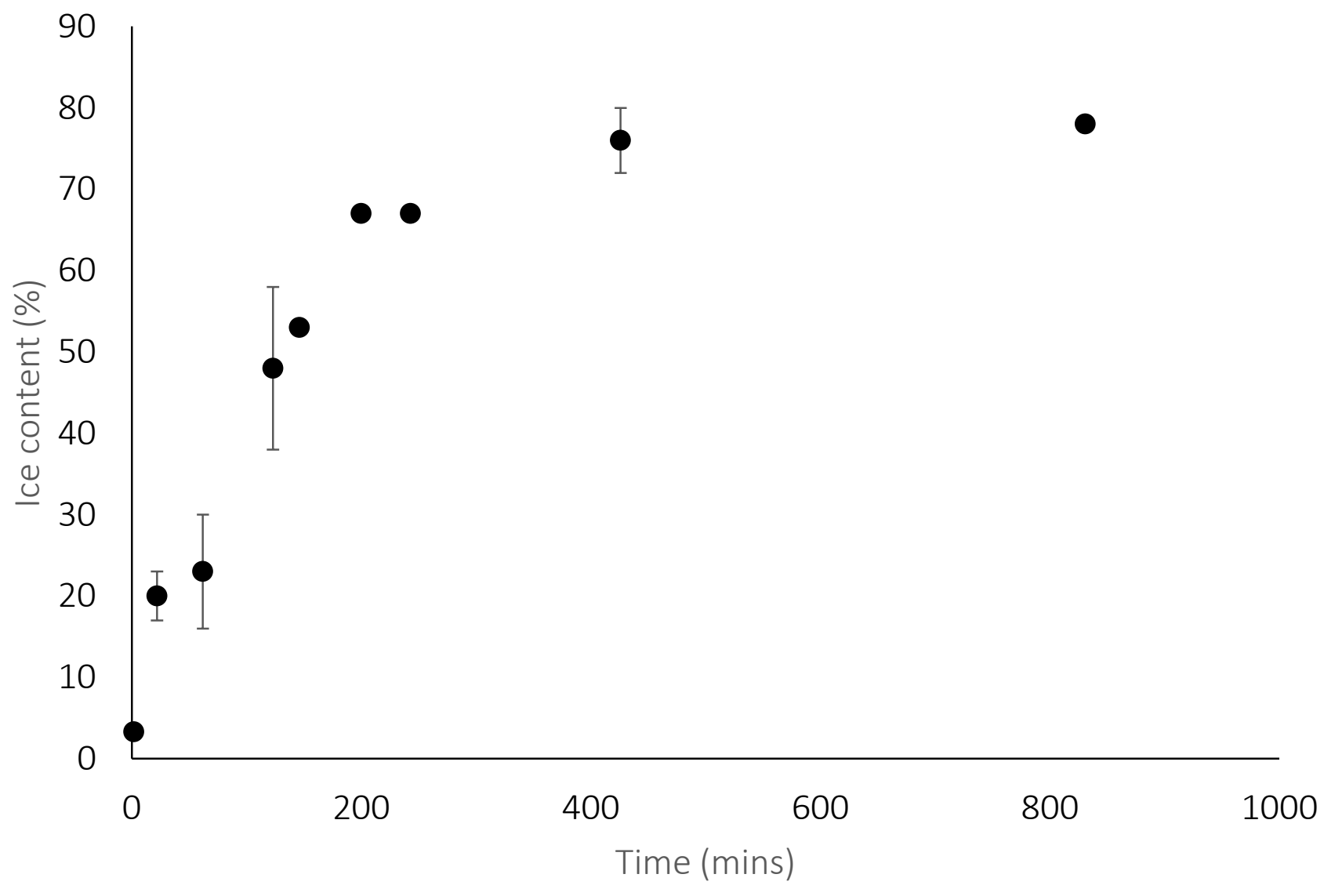




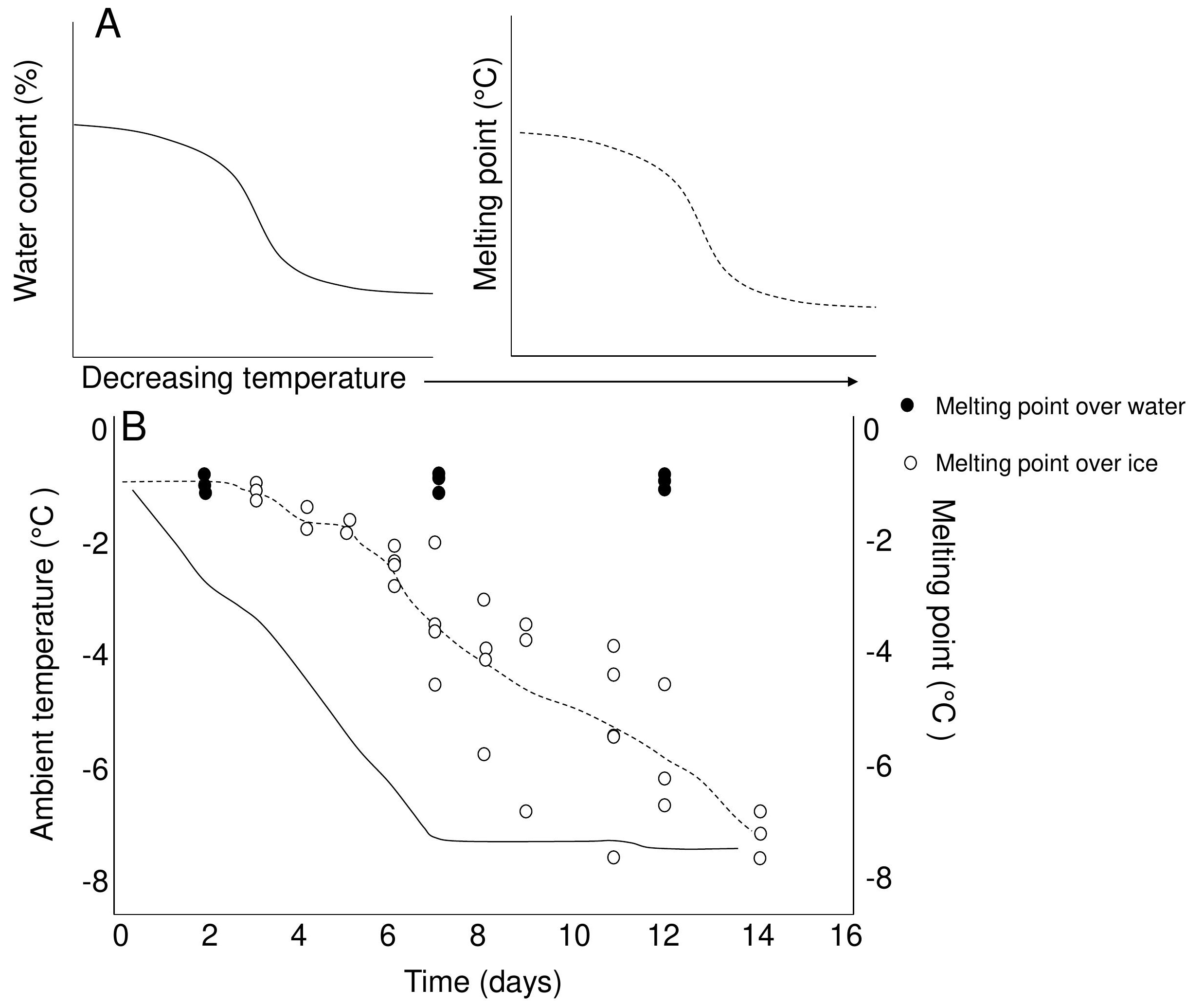




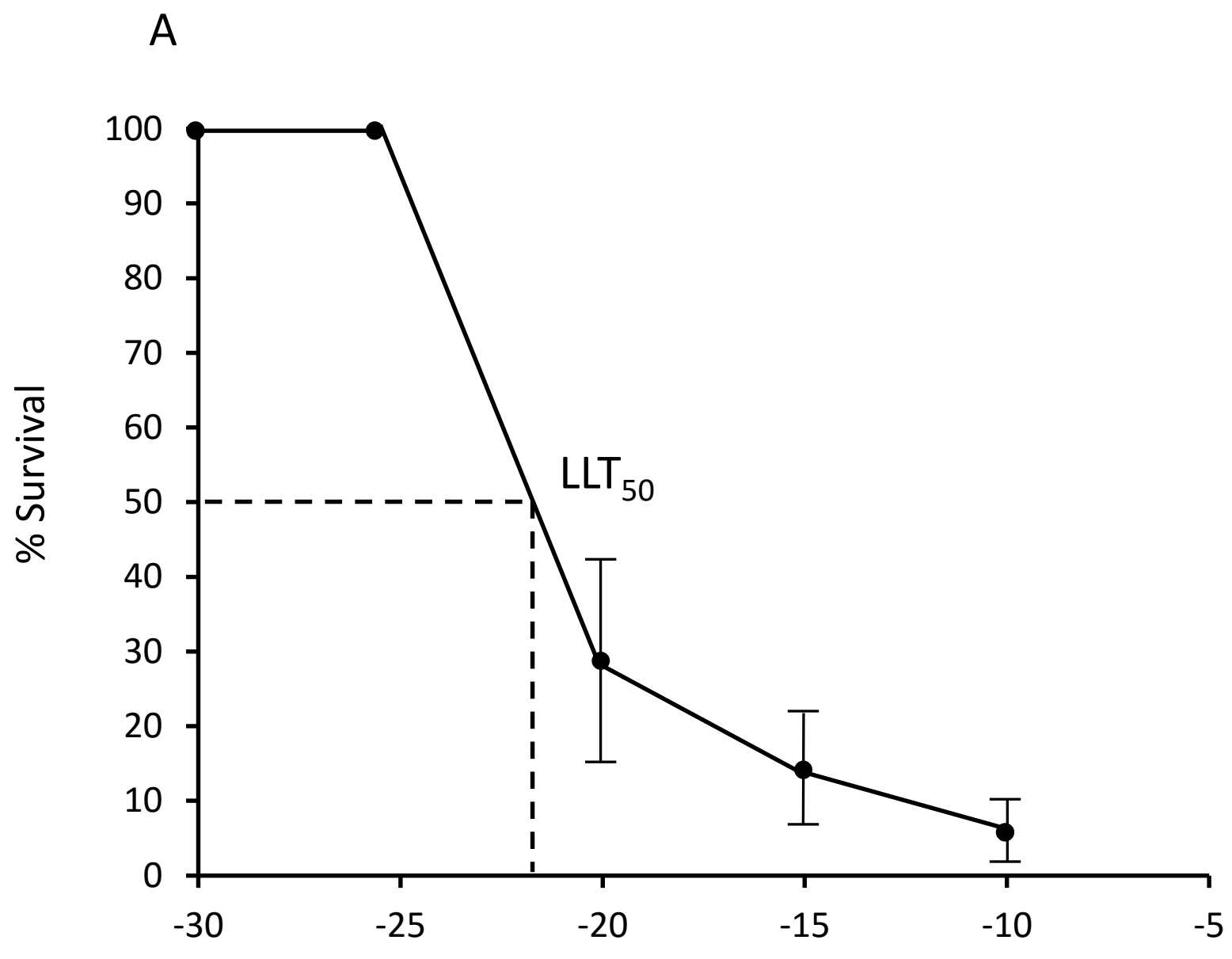

Temperature $\left({ }^{\circ} \mathrm{C}\right)$

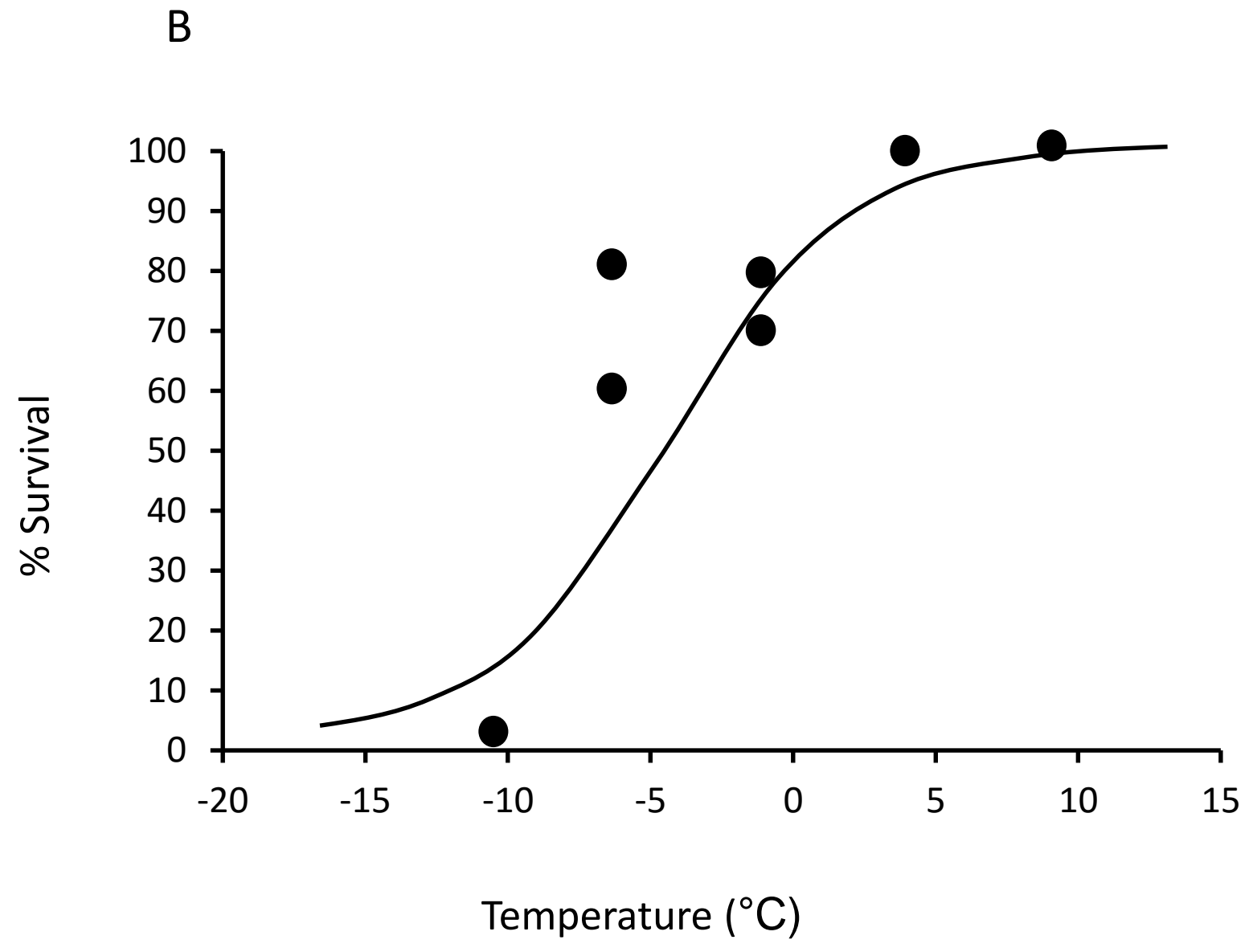




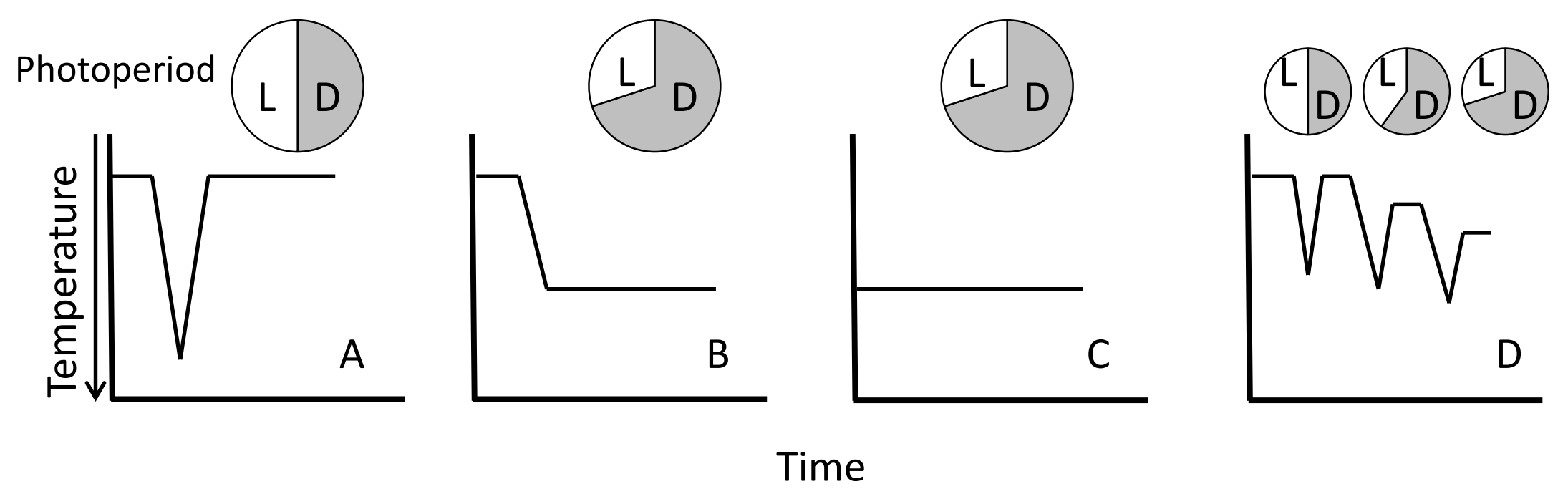


A
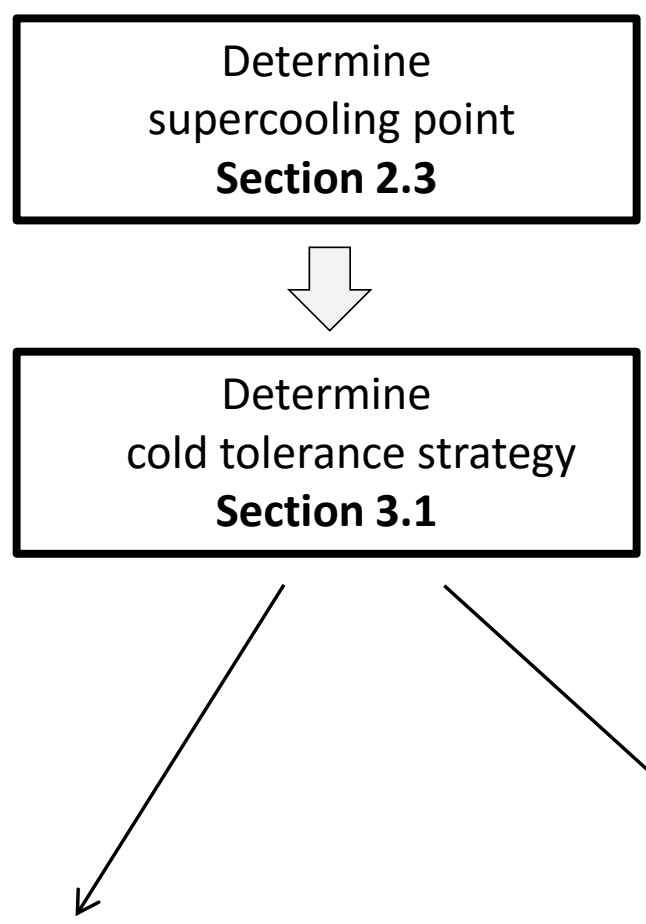

Chill-susceptible

Freeze-tolerant

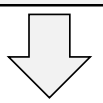

Determine lower lethal temperature Section 4.1

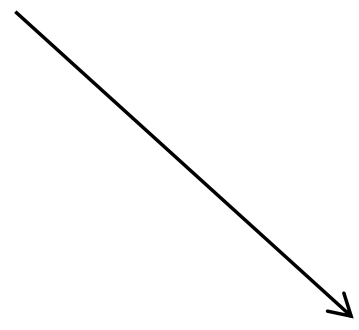

B

Determine critical thermal minimum

Section 5.1

Investigate chill coma recovery

Section 5.2

Freeze-avoidant

Investigate effects of

plasticity on cold tolerance

Section 6.5

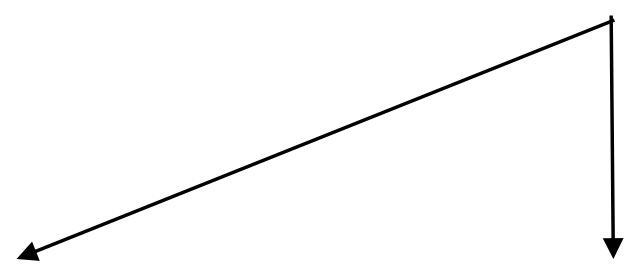

Assess cold tolerance in context of overwintering potential

(e.g. comparisons to ambient temperatures)
Compare cold tolerance among groups (See also Table 5 for additional metrics) 